\title{
Checks of integrality properties in topological strings
}

\author{
A. Mironov, ${ }^{a, b, c, f}$ A. Morozov, ${ }^{b, c, f}$ An. Morozov, ${ }^{b, c, d, f}$ P. Ramadevi, ${ }^{e}$ \\ Vivek Kumar Singh ${ }^{e}$ and A. Sleptsov ${ }^{b, c, d, f}$
}

${ }^{a}$ Theory Department, Lebedev Physics Institute, Leninsky Prospekt, Moscow, 119991 Russia

${ }^{b}$ ITEP,

Bol. Cheremushkinskaya, Moscow, 117218 Russia

${ }^{c}$ Institute for Information Transmission Problems, Bol. Karetny, Moscow, 127994 Russia

${ }^{d}$ Laboratory of Quantum Topology, Chelyabinsk State University, Bratiev Kashirinykh St., Chelyabinsk, 454001 Russia

${ }^{e}$ Department of Physics, Indian Institute of Technology Bombay, Powai, Mumbai, 400076 India

${ }^{f}$ National Research Nuclear University MEPhI, Kashirskoe Highway, Moscow, 115409 Russia

E-mail: mironov@lpi.ru, morozov@itep.ru, andrey.morozov@itep.ru, ramadevi@phy.iitb.ac.in, vivek.bhu2009@gmail.com, sleptsov@itep.ru

ABSTRACT: Tests of the integrality properties of a scalar operator in topological strings on a resolved conifold background or orientifold of conifold backgrounds have been performed for arborescent knots and some non-arborescent knots. The recent results on polynomials for those knots colored by $\mathrm{SU}(N)$ and $\mathrm{SO}(N)$ adjoint representations [1] are useful to verify Marino's integrality conjecture up to two boxes in the Young diagram. In this paper, we review the salient aspects of the integrality properties and tabulate explicitly for an arborescent knot and a link. In our knotebook website, we have put these results for over 100 prime knots available in Rolfsen table and some links. The first application of the obtained results, an observation of the Gaussian distribution of the LMOV invariants is also reported.

KEYworDS: Chern-Simons Theories, Topological Strings

ARXIV EPRINT: 1702.06316 


\section{Contents}

1 Introduction 1

2 LMOV conjecture: integrality conditions $\quad 6$

2.1 Integrality conjecture in the HOMFLY case 6

$\begin{array}{lll}2.2 & \text { Integrality conjectures in the Kauffman case } & 9\end{array}$

3 Colored polynomials for arborescent knots 12

$\begin{array}{ll}3.1 & \text { Inclusive Racah matrices for 3-strand braids } \\ \end{array}$

$\begin{array}{ll}3.2 & \text { Highest weight method } \\ \end{array}$

$\begin{array}{lll}3.3 & \text { Eigenvalue hypothesis } & 14\end{array}$

$\begin{array}{ll}3.4 & \text { Sum over paths for fundamental representations and cabling } \\ \end{array}$

$\begin{array}{ll}3.5 & \text { Two bridge and other arborescent (double-fat) knots } \\ \end{array}$

$\begin{array}{ll}3.6 \mathcal{S} \text { and } \mathcal{\mathcal { S }} \text { from exclusive Racah } & 14\end{array}$

$\begin{array}{lll}3.7 & \text { Families of arborescent knots } & 15\end{array}$

$\begin{array}{lll}3.8 & \text { Universal knot polynomials } & 15\end{array}$

$\begin{array}{ll}3.9 \text { A collection of colored knot polynomials } & 15\end{array}$

4 Tests of integrality conjectures $\quad 16$

$\begin{array}{lll}4.1 & \mathrm{SU}(N) \text { Chern-Simons } & 16\end{array}$

$\begin{array}{lll}4.2 & \mathrm{SO} / \text { Sp Chern-Simons } & 25\end{array}$

$\begin{array}{lll}4.3 & \text { Link polynomials } & 27\end{array}$

4.4 Racah matrices for links 28

5 Gaussian distributions of the LMOV numbers $N \quad 30$

6 Conclusion $\quad 31$

\section{Introduction}

Topological string duality conjectures put forth by Gopakumar-Vafa [2, 3] and OoguriVafa [4] relates $\mathrm{U}(N)$ Chern-Simons theory on $S^{3}$ to topological string theory on resolved conifold. This has led to rewrite suitable combinations of Chern-Simons knot polynomials [5-14] as reformulated invariants possessing integrality structures [15-18, 69] famously known as LMOV condition (see also [19-21] for the latest development). These integer invariants count the BPS states (spectra of M2 branes ending on M5 branes in M-theory compactified on the conifold $[2,3]$ ). These integers determine the oriented topological string amplitudes. The challenge to obtain the integers needs polynomial form of unreduced colored HOMFLY-PT for any knot/link. In fact, a recent breakthrough [22-24]-[1,35] enabled evaluation of colored HOMFLY-PT polynomials. Our knotebook website [36] which gets 
updated periodically gives the list of knots for which polynomials are obtained. Thus we can indirectly determine the BPS integers for such knots and thus verify the integrality structures within topological string duality context.

The LMOV $[4,18]$ integrality condition is much stronger than the integrality of colored HOMFLY-PT and Kauffman knot polynomials. That is, in suitable variables $(q=$ $\exp \left(\frac{2 \pi i}{k+N}\right)$ and $\left.A=q^{N}\right)$, the expectation values of Wilson loop operators

$$
P_{R}^{\mathcal{L}}(A, q)=\left\langle\operatorname{Tr}_{R} T \exp \left(\oint_{\mathcal{L}} \mathcal{A}\right)\right\rangle
$$

in $\mathrm{SU}(N)$ (colored HOMFLY-PT) or $\mathrm{SO}(N+1)$ (colored Kauffman) Chern-Simons theories are Laurent polynomials with integer coefficients. There was no topological arguments to justify these integers. The categorification technique introduced by KhovanovRozansky [37-57] interprets these integers as dimension of doubly graded vector space. It is still a challenging question to find the connection of such a categorification approach and the conventional Reshetikhin-Turaev (RT) formalism [58-63]-[80]. In refs. [81-85], these integers in Jones polynomials are interpreted as counting solutions of Hitchin equation in a four dimensional gauge theory for a given instanton number.

There is an elegant way of writing expectation value of Ooguri-Vafa scalar operator for knots in topological strings [4] using plethystic exponential of a spectrum generating function (SGF) known as index. Technically, if a Hilbert space has a SGF

$$
\operatorname{Ind}_{H}(t)=\sum_{i} \tilde{N}_{i} t^{i}
$$

then, the Fock space has a single state (vacuum) at the zeroth level, $M_{1}=\tilde{N}_{1}$ states at the first level, $M_{2}=\tilde{N}_{2}+\frac{1}{2} \tilde{N}_{1}\left(\tilde{N}_{1}+1\right)$ states at the second level, $M_{3}=\tilde{N}_{3}+\tilde{N}_{2} \tilde{N}_{1}+$ $\frac{1}{6} \tilde{N}_{1}\left(\tilde{N}_{1}+1\right)\left(\tilde{N}_{1}+1\right)$ at the third, and so on, and the SGF in the Fock space is the plethystic exponential of "the free energy" (of SGF) in the Hilbert space:

$$
\operatorname{Ind}_{F}(t)=\sum_{I} M_{I} t^{I}=\prod_{i} \frac{1}{\left(1-t^{i}\right)^{\tilde{N}_{i}}}=\exp \sum_{d=1} \frac{\operatorname{Ind}_{H}\left(t^{d}\right)}{d} .
$$

Thus, if some quantity is supposed to have an interpretation as an SGF, its plethystic logarithm should also resemble an SGF: possess integrality properties. The original OoguriGopakumar-Vafa conjecture [2-4] for knot polynomials reflected the old belief that they are actually characters, and the plethysm operation is well known to act naturally on the characters [86] (physically the plethysm operation in the conjecture is related with the Schwinger mechanism of brane creation [2, 3]). For example, the generating function of quantum dimensions (in knot theory these are unreduced HOMFLY polynomials for the unknot) is

$$
\begin{aligned}
Z_{\mathrm{OV}}^{\mathrm{unknot}}\{A, q \mid \bar{p}\} & =\sum_{R} \operatorname{dim}_{R}(A, q) \cdot \chi_{R}\{\bar{p}\}=\sum_{R} \chi_{R}\left(p^{*}\right) \chi_{R}(p)=\exp \left(\sum_{d} \frac{p_{d}^{*} \bar{p}_{d}}{d}\right) \\
& =\exp \left(\sum_{d} \frac{1}{d} \widehat{A d}_{d}\left(p_{1}^{*}\right) \widehat{A d}_{d}\left(\bar{p}_{1}\right)\right)=\exp \left(\sum_{d} \frac{p_{1}^{*}\left(A^{d}, q^{d}\right)}{d} \widehat{A d}_{d}\left(\bar{p}_{1}\right)\right),
\end{aligned}
$$


where the sum runs over all Young diagrams $R$ ("colors"), $\chi_{R}$ are the Schur functions of time variables $\bar{p}_{k}$ and the Adams (plethysm) operation $\widehat{A d}_{d}: p_{k} \longrightarrow p_{k d}$ acts at the topological locus $[73-76,87]$ by raising the power of parameters:

$$
\widehat{A d}_{d}\left(p_{k}^{*}\right)=p_{k d}^{*}=\frac{A^{k d}-A^{-k d}}{q^{k d}-q^{k d}}=p_{k}^{*}\left(A^{d}, q^{d}\right) .
$$

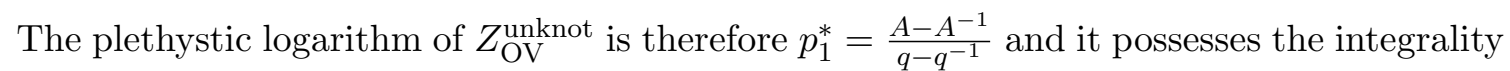
property:

$$
\left(q-q^{-1}\right) \cdot p_{1}^{*}=A-A^{-1}
$$

is a Laurent polynomial in variables $A$ and $q$ with integer coefficients for any knot. In this case of unknot, it is actually independent of $q$. The claim is that such an integrality property is true for plethystic logarithms for the Ooguri-Vafa generating functions of colored HOMFLY for all knots. In particular, it suggests that free energies have only the first order poles in the Planck constant $\hbar=\log q$ which is expected for the partition function but not so obvious for unreduced knot polynomials $\left(P_{R} \sim \hbar^{-|R|}\right)$.

As follows from (1.4), the plethystic transform exactly compensates the deviation, of the dimension $d_{R}$ from $\frac{d_{1}^{|R|}}{|R| !}$ (both classical and quantum dimensions). Further the deviation of the HOMFLY polynomial of general knots from the quantum dimension, i.e. non-classical nature of cabling, is measured by the Ooguri-Vafa polynomials $f_{R}$ (reformulated invariants). Basically, these are homogeneous polylinear combinations

$$
f_{R}=H_{R}+\sum_{I} c_{I} \prod_{i \in I} \widehat{A d}_{n_{i}}\left(H_{R_{i}}\right)
$$

with $\sum_{i} n_{i}\left|R_{i}\right|=|R|$ and all $\left|R_{i}\right|<|R|$, which vanish if all HOMFLY polynomials are substituted by the dimensions, $H_{R} \longrightarrow d_{R}$, e.g. $f_{[2]}=H_{[2]}-\frac{1}{2} H_{[1]}^{2}-\frac{1}{2} \widehat{A d}_{2}\left(H_{1}\right)$. This property defines them up to triangular transforms, and they automatically have only the first-order poles in $\{q\}\left(=q-q^{-1}\right)$.

While similarity between knot polynomials and characters of an infinite-dimensional algebra is still a plausible conjecture (see some examples in [88]), the fact is that these averages (1.1) for arbitrary representations involves character decomposition. Further localization ideas a la [89-96] can convert these averages into a finite-dimensional matrix model integral satisfying the AMM/EO topological recursion [97-100]. This has been achieved for the torus knots $[101,102]$. There are still difficulties implementing AMM/EO topological recursion for twist knots $[103,104]$ but there is some evidence of applicability of AMM/EO recursion to the non-torus knot 41 [105].

Motivated by the t' Hooft large $N$ genus expansion (closed string partition function) for free energies in gauge theories, we do expect genus expansion in $\hbar$ at fixed t' Hooft coupling ( $A$ fixed) for logarithm of the colored HOMFLY polynomials $\ln H_{R}^{\mathcal{K}}(A, q)$. There is an alternative genus expansion [106-109] known as Hurwitz-Fourier transform in variable $\mathfrak{h}$ :

$H_{R}^{\mathcal{K}}(A, q)=\operatorname{dim}_{R}(A, q) \cdot\left(\sigma_{\square}^{\mathcal{K}}(A)\right)^{|R|} \cdot \exp \left(\sum_{\Delta} \mathfrak{h}^{|\Delta|+l(\Delta)-2} s_{\Delta}^{\mathcal{K}}\left(A, \mathfrak{h}^{2}\right) \varphi_{R}(\Delta)\right) \mathfrak{h}=\frac{\hbar}{\left(\sigma_{\square}^{\mathcal{K}}(A)\right)^{2}}$ 
where $\sigma_{\square}^{\mathcal{K}}(A)=H_{\square}^{\mathcal{K}}(q=1, A)$ are called special polynomials. This expansion results in the appearance of Hurwitz-Tau function when substituted in the Ooguri-Vafa partition function $Z_{\mathrm{OV}}^{\mathcal{K}}(A, q, \bar{p})$ [110-114]. Here the sum goes over the Young diagrams $\Delta$ with $l(\Delta)$ lines of lengths $\delta_{i}$ and the number of boxes $|\Delta|=\sum_{i}^{l(\Delta)} \delta_{i}$, while $\varphi_{R}(\Delta)$ are proportional to the characters of symmetric groups $\psi_{R}(\Delta)$ at $|R|=|\Delta|: \psi_{R}(\Delta)=z_{\Delta} d_{R} \varphi_{R}(\Delta)$, and continued to $|R|>|\Delta|$ as in [110, eq. (3)]. Here $d_{R}$ is the dimension of representation $R$ of the symmetric group $S_{|R|}$ divided by $|R|$ ! and $z_{\Delta}$ is the standard symmetric factor of the Young diagram (order of the automorphism) [115]. With this definition, the sum in (1.8) runs over all $\Delta$ such that $|\Delta| \leq|R|$, while in all sums below we use $\psi_{R}(\Delta)$ which leaves in sums only terms with $|R|=|\Delta|$. An advantage to use the expansion (1.8) is in a possibility of lifting $\varphi_{R}(\Delta)$ to a ring of cut-and-join operators $W_{\Delta}[110,111]$, while using the basis of $\psi_{R}(\Delta)$ is better for studying integrality conjectures.

This Hurwitz version of the Fourier transform in the color index $R,(1.8)$ converts the set of colored HOMFLY polynomials into a collection of generalized special polynomials $\sigma_{g \mid \Delta}^{\mathcal{K}}(A)$ [106-109]. They enter (1.8) through

$$
s_{\Delta}^{\mathcal{K}}\left(A, \mathfrak{h}^{2}\right)=\sum_{g \geq 0} \mathfrak{h}^{2 g} \sigma_{g \mid \Delta}^{\mathcal{K}}(A)
$$

Note that the free energy behaves as $\hbar^{-2}$, which is natural for $\tau$-functions.

The properties of this genus/Hurwitz expansion of individual knot polynomials did not yet gain enough attention. However, eq. (1.8) calls for study of the genus expansion of the Ooguri-Vafa partition functions and implies that a natural form for it should involve the plethystic exponential:

$$
Z_{\mathrm{OV}}^{\mathcal{K}}\{A, q \mid \bar{p}\}=\sum H_{R}^{\mathcal{K}}(A, q) \chi_{R}\{\bar{p}\}=\exp \left(\sum_{d \geq 1} \sum_{\Delta} \frac{\prod_{i=1}^{l(\Delta)}\left(q^{d \delta_{i}}-q^{-d \delta_{i}}\right)}{d} S_{\Delta}^{\mathcal{K}}\left(A^{d}, q^{d}\right) \widehat{A d}_{d}\left(\bar{p}_{\Delta}\right)\right)
$$

where $\bar{p}_{\Delta}=\prod_{i} \bar{p}_{i}^{\mu_{i}}$ and $\widehat{A d}_{d}\left(\bar{p}_{\Delta}\right)=\prod_{i} \bar{p}_{d i}^{\mu_{i}}$, where $\mu_{i}$ is equal to the number of times that the line of length $i$ is met in the Young diagram $\Delta$. In these terms, $z_{\Delta}=\prod_{i} i^{\mu_{i}} \mu_{i}$ !.

Relation between (1.10) and (1.8) is not at all naive, since the sum of logarithm is not equal to a logarithm of the sum, or the sum of genus expansions is not the same as a genus expansion of the sum. It involves generalizations of the Cauchy formula (1.4) to the generation function of the generalized Hurwitz numbers [110, 111, 116, 117]

$$
Z_{\mathrm{OV}}^{\mathcal{K}}\{A, q \mid \bar{p}\} \equiv Z_{\mathrm{Hurw}}=\sum_{R} \operatorname{dim}_{R}(A, q) \cdot \chi_{R}\{\bar{p}\} \cdot e^{\sum_{\Delta} \beta_{\Delta} \varphi_{R}(\Delta)}
$$

where $\beta_{\Delta}$ encodes knot $\mathcal{K}$ information. The $\varphi_{R}(\Delta)$ appears in the "multipoint correlators" of generalized symmetric group characters as follows:

$$
\operatorname{Hurw}_{q}\left(\Delta_{1}, \Delta_{2}, \ldots, \Delta_{m}\right)=\sum_{|R|=q} d_{R}^{2} \varphi_{R}\left(\Delta_{1}\right) \varphi_{R}\left(\Delta_{2}\right) \ldots \varphi_{R}\left(\Delta_{m}\right)
$$


Note that (1.10) uses yet another different version of genus expansion, that is, in power of $q-q^{-1}$ rather than $\hbar[106-109]$. One can rewrite the product

$$
\prod_{i=1}^{l(\Delta)}\left(q^{d \delta_{i}}-q^{-d \delta_{i}}\right)=\prod_{j=1}\left(q^{j d}-q^{-j d}\right)^{\mu_{j}}
$$

which resembles the measure for the $\beta$-ensemble [118]: $\left(x_{1}-x_{2}\right)^{\beta} \longrightarrow \prod_{i=0}^{\beta-1}\left(x_{1}-q^{2 i} x_{2}\right)$. One can also look at it as a product of $q$-numbers $\left[\delta_{i}\right]_{q^{d}}$ or $[j]_{q^{d}}^{\mu_{j}}$, which generates an additional factor of $\left(q^{d}-q^{-d}\right)^{l(\Delta)}$. Hence, the additional suppression $\hbar^{|\Delta|}$ in (1.8) disappears from (1.10).

The LMOV integrality conjecture claims that after one more Hurwitz transform of $S_{\Delta}(q, A)$ in $(1.10)$,

$$
S_{\Delta}(q, A)=\sum_{Q} \psi_{Q}(\Delta) \cdot G_{Q}(A, q)
$$

the genus expansions

$$
G_{Q}(A, q)=\sum_{g \geq 0, k} N_{Q, g, k} A^{k}\left(q-q^{-1}\right)^{2 g-2}
$$

have integer coefficients. Moreover, integers $N_{Q, g, k}$ at fixed $Q$ and $k$ actually vanish at high enough genus $g$, this is an advantage of the above mentioned invariant version of the expansion. This LMOV integrality of every term of the genus expansion is, of course, much stronger than just integrality of the entire free energy.

Note that relation (1.14) can be immediately inverted:

$$
G_{Q}(A, q)=\sum_{\Delta} \frac{1}{z_{\Delta}} \psi_{Q}(\Delta) S_{\Delta}(q, A)
$$

due to the orthogonality conditions

$$
\sum_{R} \frac{1}{z_{\Delta}} \psi_{R}(\Delta) \psi_{R}\left(\Delta^{\prime}\right)=\delta_{\Delta \Delta^{\prime}}, \quad \sum_{\Delta} \frac{1}{z_{\Delta}} \psi_{R}(\Delta) \psi_{R^{\prime}}(\Delta)=\delta_{R R^{\prime}}
$$

An important implication of the Hurwitz approach is that $Z_{\mathrm{OV}}\{\bar{p}\}$ should satisfy the AMM/OE topological recursion in $g$, and this fact was actually used in the study of proofs of LMOV relation in $[122,123]$. Such studies have not be extended to prove Marino's integrality conjectures involving Kauffman polynomials which we hope to pursue in future.

It is appropriate to mention that Marino's conjectures have been verified for some torus knots and links [124-127] and figure-eight knot [128]. Hence the main focus in this paper is to verify Marino's integrality conjectures for various arborescent knots up to 8 crossings using colored Kauffman polynomials $(\mathrm{SO}(N)$ colors up to two boxes in Young diagram) and HOMFLY polynomials for mixed $\mathrm{SU}(N)$ representations. The latter are calculated using the universal Racah matrices [1] which are known only for the arborescent knots.

Informally, arborescent knots are the ones which look like trees with two-bridge branches, i.e. by gluing together the fragments of 4-strand antiparallel braids, see [29], where they 
were named double-fat. This is what makes their knot polynomials expressible through the two simplest Racah matrices $\mathcal{S}$ and $\overline{\mathcal{S}}$ (see section 3.5 below). They are also well familiar in formal knot theory, see $[14,129,130]$ for an abstract definition and other details. The list of arborescent knots includes, in particular, all twisted, 2-bridge and pretzel knots. This means that all prime knots with up to 7 intersections are arborescent being 2-bridge. Among the knots with 8 crossings the only non-arborescent prime knot is $8_{18}$. Moving further in the Rolfsen table, among the knots with 9 crossings, only $9_{34}, 9_{39}, 9_{40}, 9_{41}$, $9_{47}, 9_{49}$ are non-arborescent. At last, among the knots with 10 crossings, $10_{100}-10_{123}$ and $10_{155}-10_{165}$ are non-arborescent (polyhedral).

We will also present the LMOV integrality structures for $\mathrm{SU}(N)$ colors up to four boxes in the Young diagram as well. It allows us to reveal a striking feature of the LMOV numbers: it turns out that, with a very high accuracy, the LMOV numbers appeared to be distributed by a Gaussian law as functions of the genus $g$ ! It is just an observation that has been tested for various knots, ${ }^{1}$ and no exception has been found so far.

In section 2, we review the exact formulation of the integrality conjectures. In section 3 , we briefly recapitulate the recent progress in knot polynomial calculus. In section 4 , we will present in detail the integrality checks for a particular knot and link. We refer the reader to our dedicated site [36] where the results for other knots are updated. In section 5 , we report on the first application of the obtained results: the Gaussian distribution of the LMOV invariants. In the concluding section 6, we summarize the results obtained.

\section{LMOV conjecture: integrality conditions}

\subsection{Integrality conjecture in the HOMFLY case}

As we explained in the introduction section, the genus expansion in knot theory is determined using gauge/string duality [2-4]. That is, U $(N)$ Chern-Simons theory on a threemanifold $S^{3}$ is equivalent to topological string theory on a Calabi-Yau manifold which is the resolution of the conifold. The expectation value of the Ooguri-Vafa scalar operator associated with knots in $S^{3}$,

$$
\mathcal{Z}_{\mathrm{SU}(N)}^{\mathcal{K}}\{A, q \mid \bar{p}\}=\sum_{R} H_{R}^{\mathcal{K}}(A, q) \cdot \chi_{R}\{\bar{p}\}
$$

which is a generating function of the unreduced HOMFLY polynomials $H_{R}^{\mathcal{K}}(A, q)$, results in $A$-model open topological string partition function. In fact the logarithm of the operator can be interpreted as "connected" correlators $f_{R}(q, A)$ as follows:

$$
\log \mathcal{Z}_{\mathrm{SU}}^{\mathcal{K}}\{A, q \mid \bar{p}\}=\sum_{R} \sum_{d=1}^{\infty} \frac{1}{d} f_{R}^{\mathcal{K}}\left(A^{d}, q^{d}\right) \cdot \widehat{A d}_{d} \chi_{R}\{\bar{p}\} .
$$

We call the new quantities $f_{R}$ plethystic transforms of the adjoint HOMFLY polynomials by the reasons explained in the introduction section. Note that, the relation (1.14) can

\footnotetext{
${ }^{1}$ We checked it for all the arborescent knots that have 3 -strand braid representation: $3_{1}, 4_{1}, 5_{1}, 5_{2}$, $6_{2}, 6_{3}, 7_{1}, 7_{3}, 7_{5}, 8_{2}, 8_{5}, 8_{7}, 8_{9}, 8_{10}, 8_{16}, 8_{17}, 8_{18}, 8_{19}, 8_{20}, 8_{21}$ (see section 3.9), for the $T[2,2 k+1]$ and $T[3,3 k+1]$ series of torus knots, and for the mutant pair $11 n 41$ and $11 n 47$.
} 
be inverted leading to constructing the inverse of plethysm transformation as performed in [17]:

$$
f_{R}^{\mathcal{K}}(A, q)=\sum_{d, m=1}(-1)^{m-1} \frac{\mu(d)}{m d} \sum_{\Delta_{1}, \ldots \Delta_{m}} \widehat{A d}_{d} \psi_{R}\left(\sum_{i=1}^{m} \Delta_{i}\right) \cdot \sum_{R_{1}, \ldots, R_{m}} \prod_{j=1}^{m} \frac{\psi_{R_{j}}\left(\Delta_{j}\right)}{z_{\Delta_{j}}} H_{R_{j}}^{\mathcal{K}}\left(A^{d}, q^{d}\right)
$$

where the sum of two Young diagrams $\Delta$ and $\Delta^{\prime}$ is the Young diagram with the lines $\left\{\delta_{i}, \delta_{i}^{\prime}\right\}$ with a proper reordering, $\widehat{A d}_{d} \Delta=\widehat{A d}_{d}\left\{\delta_{i}\right\}=\left\{d \delta_{i}\right\}$, and $\mu(d)$ is the Möbius function defined as follows: if the prime decomposition of $d$ consists of $m$ multipliers and contains non-unit multiplicities, $\mu(d)=0$, otherwise $\mu(d)=(-1)^{m}$. For representations up to four boxes in Young diagram, the explicit form of the above equation will be

$$
\begin{aligned}
f_{[1]}= & H_{[1]}(q, A) \\
f_{[2]}= & H_{[2]}(q, A)-\frac{1}{2}\left(H_{[1]}(q, A)^{2}+H_{[1]}\left(q^{2}, A^{2}\right)\right) \\
f_{\left[1^{2}\right]}= & H_{\left[1^{2}\right]}(q, A)-\frac{1}{2}\left(H_{[1]}(q, A)^{2}-H_{[1]}\left(q^{2}, A^{2}\right)\right) \\
f_{[3]}= & H_{[3]}-H_{[2]} H_{[1]}+\frac{1}{3} H_{[1]}^{3}-\frac{1}{3} H_{[1]}\left(A^{3}, q^{3}\right) \\
f_{[2,1]}= & H_{[2,1]}-H_{[2]} H_{[1]}-H_{\left[1^{2}\right]} H_{[1]}+\frac{2}{3} H_{[1]}^{3}-\frac{1}{3} H_{[1]}\left(A^{3}, q^{3}\right) \\
f_{\left[1^{3}\right]}= & H_{\left[1^{3}\right]}-H_{\left[1^{2}\right]} H_{[1]}+\frac{1}{3} H_{[1]}^{3}-\frac{1}{3} H_{[1]}\left(A^{3}, q^{3}\right) \\
f_{[4]}= & H_{[4]}-H_{[3]} H_{[1]}+H_{[2]} H_{[1]}^{2}-\frac{1}{2} H_{[2]}^{2}-\frac{1}{4} H_{[1]}^{4}-\frac{1}{2} H_{[2]}\left(A^{2}, q^{2}\right)+\frac{1}{4} H_{[1]}^{2}\left(A^{2}, q^{2}\right) \\
f_{[3,1]}= & H_{[3,1]}-H_{[3]} H_{[1]}-H_{[2,1]} H_{[1]}-H_{[2]} H_{\left[1^{2}\right]}+2 H_{[2]} H_{[1]}^{2}+H_{\left[1^{2}\right]} H_{[1]}^{2}-\frac{1}{2} H_{[2]}^{2}-\frac{3}{4} H_{[1]}^{4} \\
& +\frac{1}{2} H_{[2]}\left(A^{2}, q^{2}\right)-\frac{1}{4} H_{[1]}^{2}\left(A^{2}, q^{2}\right) \\
f_{\left[2^{2}\right]}= & H_{\left[2^{2}\right]}-H_{[2,1]} H_{[1]}+H_{[2]} H_{[1]}^{2}+H_{\left[1^{2}\right]} H_{[1]}^{2}-\frac{1}{2} H_{[2]}^{2}-\frac{1}{2} H_{\left[1^{2}\right]}^{2} \\
& -\frac{1}{2} H_{[1]}^{4}-\frac{1}{2} H_{[2]}\left(A^{2}, q^{2}\right)-\frac{1}{2} H_{\left[1^{2}\right]}\left(A^{2}, q^{2}\right)+\frac{1}{2} H_{[1]}^{2}\left(A^{2}, q^{2}\right) \\
f_{\left[2,1^{2}\right]}= & H_{\left[2,1^{2}\right]}-H_{\left[1^{3}\right]} H_{[1]}-H_{[2,1]} H_{[1]}-H_{[2]} H_{\left[1^{2}\right]}+2 H_{\left[1^{2}\right]} H_{[1]}^{2}+H_{[2]} H_{[1]}^{2} \\
& -\frac{1}{2} H_{[2]}^{2}-\frac{3}{4} H_{[1]}^{4}+\frac{1}{2} H_{\left[1^{2}\right]}\left(A^{2}, q^{2}\right)-\frac{1}{4} H_{[1]}^{2}\left(A^{2}, q^{2}\right) \\
f_{\left[1^{4}\right]}= & H_{\left[1^{4}\right]}-H_{\left[1^{3}\right]} H_{[1]}+H_{\left[1^{2}\right]} H_{[1]}^{2}-\frac{1}{2} H_{\left[1^{2}\right]}^{2}-\frac{1}{4} H_{[1]}^{4}-\frac{1}{2} H_{[2]}\left(A^{2}, q^{2}\right)+\frac{1}{4} H_{[1]}^{2}\left(A^{2}, q^{2}\right) .
\end{aligned}
$$

Now, the expansion of HOMFLY polynomial is translated into a similar expansion of the plethystic polynomials:

$$
f_{R}(q, A)=\sum_{n, k} \widetilde{\mathbf{N}}_{R, n, k} \frac{A^{n} q^{k}}{q-q^{-1}}
$$


Even though HOMFLY behaves as $1 /\left(q-q^{-1}\right)^{|R|}$, the reformulated invariant $f_{R}(q, A)$ has only singularity $1 /\left(q-q^{-1}\right)$

In fact, the only way to check this duality between Chern-Simons and topological string theories is to establish the integrality condition: the coefficients $\widetilde{\mathbf{N}}_{R, n, k}$ has to be integer in accordance with the Ooguri-Vafa conjecture [4]. Moreover, as we explained in the introduction section, one can construct even more refined integers (1.14) [16]

$$
f_{R}(q, A)=\sum_{n, k \geq 0, Q} C_{R Q} \mathbf{N}_{Q, n, k} A^{n}\left(q-q^{-1}\right)^{2 k-1}
$$

where

$$
C_{R Q}=\sum_{\Delta} \frac{1}{z_{\Delta}} \psi_{R}(\Delta) \psi_{Q}(\Delta) \frac{\prod_{i=1}^{l(\Delta)}\left(q^{\delta_{i}}-q^{-\delta_{i}}\right)}{q-q^{-1}}=\frac{1}{q-q^{-1}} \sum_{\Delta} \frac{1}{z_{\Delta}} \psi_{R}(\Delta) \psi_{Q}(\Delta)^{*} p_{\Delta}
$$

with ${ }^{*} p_{k} \equiv q^{k}-q^{-k}$. To compare this formula with (1.14), one has to use the identity

$$
\sum_{R} \psi_{R}(\Delta) \chi_{R}(p)=p_{\Delta}
$$

This matrix can be easily reversed using (1.17):

$$
\left(C^{-1}\right)_{Q R}=\sum_{\Delta} \frac{1}{z_{\Delta}} \psi_{R}(\Delta) \psi_{Q}(\Delta) \frac{q-q^{-1}}{{ }^{*} p_{\Delta}} .
$$

Let us note that there is a hierarchy of integralities: the weakest statement is the claim that the HOMFLY polynomials are integer. The next level is integrality of $\widetilde{\mathbf{N}}_{R, n, k}$, which implies that of HOMFLY, but not vice versa. However, $\widetilde{\mathbf{N}}_{R, n, k}$ are not independent: they satisfy some relations [15], while the more refined $\mathbf{N}_{R, n, k}$ are independent numbers and their integrality implies the integrality of $\tilde{\mathbf{N}}_{R, n, k}$, but not vice versa. This means that $\mathbf{N}_{R, n, k}$ are, in a sense, elementary building blocks. Their integrality from the knot theory point of view is not at all evident. Note that the BPS invariants $\mathbf{N}_{R, n, k}$ are linearly related to the Gopakumar-Vafa (open Gromov-Witten) invariants $\mathbf{n}_{\Delta, n, k}[2,3]$ :

$$
\mathbf{n}_{\Delta, n, k}=\sum_{R} \psi_{R}(\Delta) \mathbf{N}_{R, n, k}, \quad \mathbf{N}_{R, n, k}=\sum_{\Delta} \frac{1}{z_{\Delta}} \psi_{R}(\Delta) \mathbf{n}_{\Delta, n, k}
$$

and the integrality of $\mathbf{n}_{\Delta, n, k}$ follows from the integrality of $\mathbf{N}_{R, n, k}$, but not vice versa. Integrality of the coefficients $\mathbf{N}_{Q, n, k}$ was checked in [69-72], and was also generally proven in $[122,123]$. However, as an illustration, we have calculated all $\mathbf{N}_{Q, n, k}$ with $|Q| \leq 4$ for the knots in the Rolfsen table [131] given by 3-strand braids and manifestly checked their integrality. We discuss this in section 4.1.

Framing dependence. Explicit answers for the functions $f_{R}(q, A)$ and, hence, for all the integers depend on the choice of framing. Remarkably, this dependence can be described by the action of the cut-and-join operator and almost does not affect the integrality: the integers remain integers [18] at any framing with a small additional rescaling of the HOMFLY polynomials entering the definition (2.1), though the dependence of integers on the 
framing is quite weird (see examples in $[18 \text {, section } 4.3]^{2}$ ). The total framing factor contains two multipliers: $A^{p|R|}$ and $q^{2 p \varphi_{R}([2])}\left(\varphi_{R}([2])\right.$ proportional to the quadratic Casimir), where $p$ is an arbitrary integer. The first multiplier is trivial, since it is removed by the replace $\bar{p}_{k} \rightarrow A^{p} \bar{p}_{k}$ in $(2.2)$, i.e. leads to a trivial factor of $A^{p|R|}$ in $f_{R}(q, A)$. The second factor is much less trivial. What is more important, in order to preserve the integrality, one has to change the definition (2.1) making it slightly dependent on framing: one should multiply the HOMFLY polynomials entering it by an additional framing factor: $H_{R}^{\mathcal{K}}(A, q) \rightarrow(-1)^{p|R|} H_{R}^{\mathcal{K}}(A, q),[132]$.

In fact, the framing story is different for knots and links. For knots, there is a distinguished topological framing (standard framing), and we present all the answers below for this choice. For links, there is no distinguished "mutual" framing of different components of the link. Moreover, here one should additionally care that the HOMFLY and Kauffman polynomials are calculated in the same framing. Another subtlety is a factor that distinguish between the reduced and unreduced knot polynomials. While in the HOMFLY case they differ just by the corresponding quantum dimensions, the standard Kauffman polynomial of a link is related with the unreduced one by multiplying with the quantum dimension and with a factor of $A^{-2} \operatorname{lk}(\mathcal{L})$, where $\operatorname{lk}(\mathcal{L})$ is the linking number.

Note that in order to fix notation in the case of links, one can use another distinguished framing, the vertical framing, which means that all $\mathcal{R}$-matrices are generated from the universal one. This prescription fixes the notation, but it is different from the topological

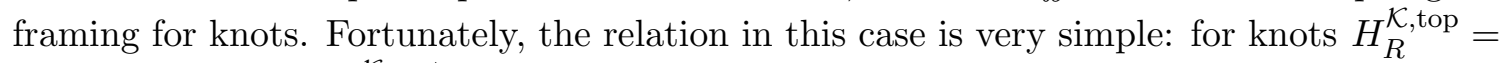
$A^{-w|R|} q^{-4 w \varphi_{R}([2])} \cdot H_{R}^{\mathcal{K}, \text { vert }}$, where $w$ is the writhe number.

\subsection{Integrality conjectures in the Kauffman case}

A natural generalization of the described correspondence is the equivalence between the $\mathrm{SO} / \mathrm{Sp}$ Chern-Simons theory and the topological string theory on an orientifold of the small resolution of the conifold [133]-[128]. In this case, the Chern-Simons partition function is associated with two types of contributions: those from oriented and non-oriented strings, the former ones coming with the degree $1 / 2:^{3}$

$$
\mathcal{Z}_{\mathrm{SO} / \mathrm{Sp}}^{\mathcal{K}}\{A, q \mid \bar{p}\}=Z_{\mathrm{no}} \sqrt{Z_{\mathrm{o}}} .
$$

Thus, one expects that

- The partition function of the oriented strings $Z_{\mathrm{o}}$ induces an integrality condition.

- The partition function of the non-oriented strings $Z_{\text {no }}$ also induces another integrality condition.

\footnotetext{
${ }^{2}$ Notice a misprint in table 8 of $[18$, section 4.3$]$ : in the second line of the table, there should be $-8-5 p-3 p^{2}$ instead of $-8-5 p-p^{2}$.

${ }^{3}$ This formula looks quite natural due to the Rudolph-Morton-Ryder theorem, [137-139]:$$
\left(K_{R}^{\mathcal{K}}\right)^{2}=H_{R, R} \quad(\bmod 2)
$$

where "mod 2" means that the integer coefficients of the Laurent polynomials in this formula are taken modulo $2, K_{R}$ is the Kauffman knot polynomial and $H_{R, S}$ denotes the HOMFLY polynomial in the composite representation [140]. In fact, the Rudolph-Morton-Ryder theorem immediately follows from the integrality conditions, see [124].
} 
We will now briefly review the necessary steps: the oriented partition function is given by the generating function of the HOMFLY polynomials in composite representations [124]:

$$
Z_{\mathrm{o}}\{A, q \mid \bar{p}\}=\sum_{R, S} H_{(R, S)}^{\mathcal{K}}(A, q) \cdot \chi_{R}\{\bar{p}\} \chi_{S}\{\bar{p}\} .
$$

Note that the sum in the second case is over a double set of Young diagrams, but there is a single set of time variables $\bar{p}$. From this partition function one builds the free energy, which is again expanded into sum over Young diagrams,

$$
\log Z_{\mathrm{o}}\{A, q \mid \bar{p}\}=\sum_{R} \sum_{d=1}^{\infty} \frac{1}{d} h_{R}^{\mathcal{K}}\left(A^{d}, q^{d}\right) \cdot \widehat{A d}_{d} \chi_{R}\{\bar{p}\}
$$

and the first few terms of expansion are

$$
\begin{aligned}
h_{[1]}^{\mathcal{K}}(A, q) & =2 H_{[1]}(A, q) \\
h_{[2]}^{\mathcal{K}}(A, q) & =2 H_{[2]}(A, q)+H_{([1],[1])}(A, q)-2\left(H_{[1]}(A, q)\right)^{2}-H_{[1]}\left(A^{2}, q^{2}\right) \\
h_{[1,1]}^{\mathcal{K}}(A, q) & =2 H_{[1,1]}(A, q)+H_{([1],[1])}(A, q)-2\left(H_{[1]}(A, q)\right)^{2}+H_{[1]}\left(A^{2}, q^{2}\right) \\
& \ldots
\end{aligned}
$$

There is a mirror symmetry under transposition of Young diagrams which relates knot polynomials as follows:

$$
H_{R^{\operatorname{tr}}}(A, q)=H_{R}\left(A,-q^{-1}\right)
$$

However its implication to $h_{R}$ is not seen. Note that the sign flip in (2.13) emerges in the course of performing the Adams transformation in (2.11).

One can again generate the refined integrality condition via

$$
h_{R}(q, A)=\sum_{n, k \geq 0, Q} C_{R Q} \hat{\mathbf{N}}_{Q, n, k}^{c=0} A^{n}\left(q-q^{-1}\right)^{2 k-1}
$$

where the superscript $c$ denotes the contribution from Riemann surfaces with $c$ crosscups $[134-136]$.

In order to calculate the non-oriented partition function, one has to calculate

$$
Z_{\mathrm{no}}=\frac{\mathcal{Z}_{\mathrm{SO} / \mathrm{Sp}}^{\mathcal{K}}\{A, q \mid \bar{p}\}}{\sqrt{Z_{\mathrm{o}}}}
$$

where the numerator is given by the generating function of the (unreduced) Kauffman polynomials

$$
\mathcal{Z}_{\mathrm{SO} / \mathrm{Sp}}^{\mathcal{K}}\{A, q \mid \bar{p}\}=\sum_{R} K_{R}^{\mathcal{K}}(A, q) \cdot \chi_{R}\{\bar{p}\}
$$

and $Z_{\mathrm{o}}$ is given by the HOMFLY polynomials in composite representations, (2.10). Hence,

$$
\log Z_{\mathrm{no}}\{A, q \mid \bar{p}\}=\log \mathcal{Z}_{\mathrm{SO} / \mathrm{Sp}}^{\mathcal{K}}\{A, q \mid \bar{p}\}-\frac{1}{2} \log Z_{\mathrm{o}}\{A, q \mid \bar{p}\}=\sum_{R} \sum_{d \geq 1, \text { odd }}^{\infty} \frac{1}{d} g_{R}^{\mathcal{K}}\left(A^{d}, q^{d}\right) \cdot \widehat{A d}_{d} \chi_{R}\{\bar{p}\}
$$


with the first terms of expansion being

$$
\begin{aligned}
g_{[1]}^{\mathcal{K}}(A, q) & =K_{[1]}(A, q)-H_{[1]}(A, q) \\
g_{[2]}^{\mathcal{K}}(A, q) & =K_{[2]}(A, q)-\frac{1}{2}\left(K_{[1]}(A, q)\right)^{2}-H_{[2]}(A, q)+\left(H_{[1]}(A, q)\right)^{2}-\frac{1}{2} H_{([1],[1])}(A, q) \\
g_{[1,1]}^{\mathcal{K}}(A, q) & =K_{[1,1]}(A, q)-\frac{1}{2}\left(K_{[1]}(A, q)\right)^{2}-H_{[1,1]}(A, q)+\left(H_{[1]}(A, q)\right)^{2}-\frac{1}{2} H_{([1],[1])}(A, q)
\end{aligned}
$$

and the integrality condition

$$
g_{R}(q, A)=\sum_{n, k \geq 0, Q} C_{R Q}\left(\hat{\mathbf{N}}_{Q, n, k}^{c=1} A^{n}\left(q-q^{-1}\right)^{2 k}+\hat{\mathbf{N}}_{Q, n, k}^{c=2} A^{n}\left(q-q^{-1}\right)^{2 k+1}\right)
$$

The above discussion for knots can be extended to two component links. The relevant operator for these links will be

$$
\begin{aligned}
Z_{\mathrm{o}}\{A, q \mid p, \bar{p}\} & =\sum_{R, S} H_{\left(R_{1}, S_{1}\right)\left(R_{2}, S_{2}\right)}^{\mathcal{L}}(A, q) \cdot \chi_{R_{1}}\{p\} \chi_{S_{1}}\{p\} \cdot \chi_{R_{2}}\{\bar{p}\} \chi_{S_{2}}\{\bar{p}\} \\
\log Z_{\mathrm{o}}\{A, q \mid p, \bar{p}\} & =\sum_{R} \sum_{d=1}^{\infty} \frac{1}{d} h_{R_{1}, R_{2}}^{\mathcal{L}}\left(A^{d}, q^{d}\right) \cdot \widehat{A d}_{d} \chi_{R_{1}}\{p\} \cdot \widehat{A d}_{d} \chi_{R_{2}}\{\bar{p}\}
\end{aligned}
$$

and

$$
\begin{aligned}
\mathcal{Z}_{\mathrm{SO} / \mathrm{Sp}}^{\mathcal{L}}\{A, q \mid p, \bar{p}\} & =\sum_{R_{1}, R_{2}} K_{R_{1}, R_{2}}^{\mathcal{L}}(A, q) \cdot \chi_{R_{1}}\{p\} \chi_{R_{2}}\{\bar{p}\} \\
\log Z_{\mathrm{no}}\{A, q \mid p, \bar{p}\} & =\log \mathcal{Z}_{\mathrm{SO} / \mathrm{Sp}}^{\mathcal{L}}\{A, q \mid p, \bar{p}\}-\frac{1}{2} \log Z_{\mathrm{o}}\{A, q \mid p, \bar{p}\} \\
& =\sum_{R} \sum_{d \geq 1, \text { odd }}^{\infty} \frac{1}{d} g_{R_{1}, R_{2}}^{\mathcal{K}}\left(A^{d}, q^{d}\right) \cdot \widehat{A d}_{d} \chi_{R_{1}}\{p\} \cdot \widehat{A d}_{d} \chi_{R_{2}}\{\bar{p}\}
\end{aligned}
$$

so that the explicit form for oriented invariants $h_{R_{1}, R_{2}}$ for some representations are

$$
\begin{aligned}
h_{[1],[1]}^{\mathcal{L}}= & 2 H_{[1],[1]}^{\mathcal{L}}+2 H_{[1],[1]}^{\overline{\mathcal{L}}}-4 H_{[1]}^{\mathcal{K}_{1}} H_{[1]}^{\mathcal{K}_{2}} \\
h_{[2],[1]}^{\mathcal{L}}= & 2 H_{[2],[1]}^{\mathcal{L}}+2 H_{[2],[1]}^{\overline{\mathcal{L}}}+2 H_{([1],[1]),[1]}^{\mathcal{L}}-4 H_{[1],[1]}^{\mathcal{L}} H_{[1]}^{\mathcal{K}_{1}}-4 H_{[1],[1]}^{\overline{\mathcal{L}}} H_{[1]}^{\mathcal{K}_{1}} \\
& -4 H_{[2]}^{\mathcal{K}_{1}} H_{[1]}^{\mathcal{K}_{2}}-2 H_{([1],[1])}^{\mathcal{K}_{1}} H_{[1]}^{\mathcal{K}_{2}}+8\left(H_{[1]}^{\mathcal{K}_{1}}\right)^{2} H_{[1]}^{\mathcal{K}_{2}} \\
h_{[1,1],[1]}^{\mathcal{L}}= & 2 H_{([1,1],[1]}^{\mathcal{L}}+2 H_{[1,1],[1]}^{\mathcal{\mathcal { L }}}+2 H_{([1],[1]),[1]}^{\mathcal{L}}-4 H_{[1],[1]}^{\mathcal{L}} H_{[1]}^{\mathcal{K}_{1}}-4 H_{[1],[1]}^{\overline{\mathcal{L}}} H_{[1]}^{\mathcal{K}_{1}} \\
& -4 H_{[1,1]}^{\mathcal{K}_{1}} H_{[1]}^{\mathcal{K}_{2}}-2 H_{([1],[1])}^{\mathcal{K}_{1}} H_{[1]}^{\mathcal{K}_{2}}+8\left(H_{[1]}^{\mathcal{K}_{1}}\right)^{2} H_{[1]}^{\mathcal{K}_{2}}
\end{aligned}
$$


and similarly

$$
\begin{aligned}
g_{[1],[1]}^{\mathcal{L}}= & K_{[1],[1]}^{\mathcal{L}}-K_{[1]}^{\mathcal{K}_{1}} K_{[1]}^{\mathcal{K}_{2}}-H_{[1],[1]}^{\mathcal{L}}-H_{[1],[1]}^{\overline{\mathcal{L}}}+2 H_{[1]}^{\mathcal{K}_{1}} H_{[1]}^{\mathcal{K}_{2}} \\
g_{[2],[1]}^{\mathcal{L}}= & K_{[2],[1]}^{\mathcal{L}}-H_{[2],[1]}-H_{[2],[1]}^{\mathcal{L}}-H_{[2],[1]}^{\mathcal{\mathcal { L }}}-K_{[1],[1]}^{\mathcal{L}} K_{[1]}^{\mathcal{K}_{1}}-K_{[2]}^{\mathcal{K}_{1}} K_{[1]}^{\mathcal{K}_{2}}+2 H_{[1],[1]}^{\mathcal{L}} H_{[1]}^{\mathcal{K}_{1}} \\
& +2 H_{[1],[1]}^{\mathcal{\mathcal { \mathcal { L } }}} H_{[1]}^{\mathcal{K}_{1}}+2 H_{[2]}^{\mathcal{K}_{1}} H_{[1]}^{\mathcal{K}_{2}}+H_{([1],[1])}^{\mathcal{K}_{1}} H_{[1]}^{\mathcal{K}_{2}}+\left(K_{[1]}^{\mathcal{K}_{1}}\right)^{2} K_{[1]}^{\mathcal{K}_{2}}-4\left(H_{[1]}^{\mathcal{K}_{1}}\right)^{2} H_{[1]}^{\mathcal{K}_{2}} \\
g_{[1,1],[1]}^{\mathcal{L}}= & K_{[1,1],[1]}^{\mathcal{L}}-H_{[1,1],[1]}-H_{[1,1],[1]}^{\mathcal{L}}-H_{[1,1],[1]}^{\mathcal{\mathcal { L }}}-K_{[1],[1]}^{\mathcal{L}} K_{[1]}^{\mathcal{K}_{1}}-K_{[1,1]}^{\mathcal{K}_{1}} K_{[1]}^{\mathcal{K}_{2}}+2 H_{[1],[1]}^{\mathcal{L}} H_{[1]}^{\mathcal{K}_{1}} \\
& +2 H_{[1],[1]}^{\mathcal{\mathcal { \mathcal { L } }}} H_{[1]}^{\mathcal{K}_{1}}+2 H_{[1,1]}^{\mathcal{K}_{1}} H_{[1]}^{\mathcal{K}_{2}}+H_{([1],[1]}^{\mathcal{K}_{1}} H_{[1]}^{\mathcal{K}_{2}}+\left(K_{[1]}^{\mathcal{K}_{1}}\right)^{2} K_{[1]}^{\mathcal{K}_{2}}-4\left(H_{[1]}^{\mathcal{K}_{1}}\right)^{2} H_{[1]}^{\mathcal{K}_{2}}
\end{aligned}
$$

where $\mathcal{K}_{1}$ and $\mathcal{K}_{2}$ are the components of the link and $\overline{\mathcal{L}}$ denotes the link obtained from $\mathcal{L}$ by reversing the orientation of one of its components. These expansions are again related to the two corresponding integrality conditions:

$$
h_{R_{1}, R_{2}}(q, A)=\sum_{n, k \geq 0, Q_{1}, Q_{2}} C_{R_{1} Q_{1}} C_{R_{2} Q_{2}} \hat{\mathbf{N}}_{Q_{1}, Q_{2}, n, k}^{c=0} A^{n}\left(q-q^{-1}\right)^{2 k}
$$

and

$g_{R_{1}, R_{2}}(q, A)=\sum_{n, k \geq 0, Q_{1}, Q_{2}} C_{R_{1} Q_{1}} C_{R_{2} Q_{2}}\left(\hat{\mathbf{N}}_{Q_{1}, Q_{2}, n, k}^{c=1} A^{n}\left(q-q^{-1}\right)^{2 k+1}+\hat{\mathbf{N}}_{Q_{1}, Q_{2}, n, k}^{c=2} A^{n}\left(q-q^{-1}\right)^{2 k+2}\right)$.

Note that, in the case of link, $h$ and $g$ have to contain additional degrees of $q-q^{-1}$ at $q \rightarrow 1$ as compared with the knot case. The integrality expansions reviewed for unoriented topological string amplitudes were conjectured [124] and verified for $(2,2 m+1)$ torus knots. Now with our recent advances in evaluation of colored knot polynomials for adjoint representations for arborescent knots and non-arborescent knots obtained from three strand braids, [29]-[1, 35], we could provide further evidence for the conjecture.

The main goal of the present paper is to determine the coefficients $N$ for a wide class of knots/links and check their integrality properties. Our results for many knots and links can be considered as a direct continuation of the appendix from [77] and especially of appendix B from [128] for figure-eight knot where the theory is presented in detail with relevant references.

In the following section we will present briefly various methods useful in the evaluation of colored polynomials.

\section{Colored polynomials for arborescent knots}

The colored HOMFLY polynomials are well defined quantities. If a link/knot is presented as the closure of a braid, then the HOMFLY polynomial is a $q^{\rho}$-weighted trace of a product of quantum $\mathcal{R}$-matrices at the intersections of strands of the braid [58-63]. In the modern version of the RT formalism [73-76]-[80], one uses the $\mathcal{R}$-matrices acting in the space of intertwining operators. Actually this defines the HOMFLY polynomial up to an overall 
framing factor. For knots there is a distinguished choice of framing called the topological framing which is independent of framing number. Remarkably, such a framing choice is not necessary for LMOV integrality structure. These properties hold for other framings where we add a suitable U(1) invariant with suitable U(1) charges [18]. However, for links the distinguished framing does not exist. Moreover, there is an additional ambiguity in HOMFLY depending on mutual orientation of components.

Despite these constraints, the colored HOMFLY polynomials are very difficult to evaluate, and we have very limited success in this direction for arborescent knots and links. The main barrier in obtaining polynomial form is the absence of $\mathrm{SU}(N)$ Racah matrices in quantum group theory. Finding these Racah matrices for arbitrary representation gets especially difficult in the case of non-trivial multiplicities (i.e. for non-rectangular Young diagrams and outside the $E_{8}$-sector [141]). Such representations with non-trivial multiplicity plays a crucial role in distinguishing mutant knot pairs [29]-[35]. In the following subsection, we will briefly review various methods leading to knot polynomials.

\subsection{Inclusive Racah matrices for 3-strand braids}

The brute force application of the modern RT formalism a la [73-76]-[80] requires knowledge of the matrices $\mathcal{R}_{a, a+1}$ acting at the crossing of adjacent strands $a$ and $a+1$ in the braid. While one of them, say $\mathcal{R}_{12}$, can be diagonalized and has very simple eigenvalues, which are just exponentials of quadratic Casimir eigenvalues $\varkappa_{Y}$ [87], the others are not diagonal and are obtained by conjugation with additional mixing matrices. In particular, $\mathcal{R}_{23}=\mathcal{U} \mathcal{R}_{12} \mathcal{U}^{\dagger}$, where $\mathcal{U}$ is a Racah matrix converting an intertwiner $(R \otimes R) \otimes R \longrightarrow Q \in R^{\otimes 3}$ into $R \otimes(R \otimes R) \longrightarrow Q$. It is a matrix acting in the space of representations $Y \in R^{\otimes 2}$. Thus the knowledge of the inclusive Racah matrix, i.e. a collection of Racah matrices for all $Q \in R^{\otimes 3}$ is sufficient for performing the 3-strand braid calculations. Going beyond three strand braid required determining a wider class of inclusive Racah matrices which is tedious.

\subsection{Highest weight method}

This method gives a straightforward evaluation of mixing matrices which requires compar-

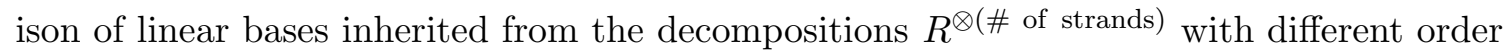
of brackets, like $R^{\otimes 2} \otimes R$ and $R \otimes R^{\otimes 2}$. Literally, if the vector spaces are associated with particular representations, this comparison gives the Clebsh-Gordon coefficients. In order to get the Racah matrices, the simplest way is to look just at the highest weight vectors as elements in the abstract Verma modules. This formalism is successfully developed in [31] and [33] and has already allowed us to find the inclusive Racah matrices for $R=[2,2]$ and even $R=[3,1]$. In combination with the differential expansion method [142-150], this provides extensions to other rectangular representations. Further progress (for other nonrectangular representations) is expected after developing the $\Delta$-technique briefly outlined in [33]. We are presently extending the work [77] investigating the highest weight method to determine polynomials of knots obtained from four or more strands carrying symmetric representation. 
Even though the method is straightforward and very successful, the calculations become cumbersome as we increase the number of strands beyond three strands.

\subsection{Eigenvalue hypothesis}

The most interesting method is the eigenvalue hypothesis [78] saying that the entries of Racah matrix are actually made from the known eigenvalues $\pm q^{\varphi_{Y}([2])}$ of $\mathcal{R}$-matrix for all representations $Y \in R^{\otimes 2}$ (the sign depends on belonging to the symmetric or antisymmetric squares). Explicit formulas are currently known up to the size $6 \times 6$ (see $[1,78]$ and [35]), while for $R=[3,1]$ Racah matrices can be $20 \times 20$. Still, most of constituents of the inclusive Racah matrices are small, and the use of eigenvalue hypothesis is practically very convenient even in its present form. However there are conceptual questions [151] that still need to be resolved within this method.

\subsection{Sum over paths for fundamental representations and cabling}

A natural way is to represent $\mathcal{R}$-matrices in the space of paths in the representation tree, which leads to a peculiar sum-over-paths formulation, at least, for the fundamental HOMFLY [79]. Then, the cabling method can be applied to extract the colored HOMFLY polynomials [80]. This method turns out to be rather powerful and calculations involving 12-strands determine [21]-colored HOMFLY polynomials for some 4-strand knots, and [31]or [22]-colored HOMFLY polynomials for the 3 -strand braids.

\subsection{Two bridge and other arborescent (double-fat) knots}

A big class, the arborescent knots [14, 129, 130], which dominate in the Rolfsen table of knots with low crossing numbers, has a peculiar double-fat realization [29], which expresses their HOMFLY polynomials through just two exclusive Racah matrices $\mathcal{S}$ : $\{(R \otimes R) \otimes \bar{R} \longrightarrow R\} \longrightarrow\{R \otimes(R \otimes \bar{R}) \longrightarrow R\}$ and $\overline{\mathcal{S}}:\{(R \otimes \bar{R}) \otimes R \longrightarrow R\} \longrightarrow\{R \otimes$ $(\bar{R} \otimes R) \longrightarrow R\}$. The term exclusive refers to selecting just one particular representation $R$ from the product $R^{\otimes 2} \otimes \bar{R}$. Exclusive is, of course, much simpler than inclusive; however, involvement of the conjugate representation (inverted strand direction), is a considerable complication. The matrices $\mathcal{S}$ and $\overline{\mathcal{S}}$ are known for all symmetric (and antisymmetric) representations $R$ [26] and [152] and, by an outstanding effort, for $R=[2,1]$ [153].

\section{6 $\mathcal{S}$ and $\overline{\mathcal{S}}$ from exclusive Racah}

A much simpler way to obtain $\mathcal{S}$ and $\overline{\mathcal{S}}$ for non-symmetric representations was suggested in [35]. Namely, the exclusive Racah matrices $\mathcal{S}$ were extracted from the HOMFLY polynomials of the double evolution family [144, 145] of 3-strand braids (which were evaluated with the known inclusive Racah matrices) in the following way: the same family can be presented as an arborescent family (of the pretzel knots), hence, its HOMFLY polynomials can be presented in the form involving the exclusive matrices in such a way that $\mathcal{S}$ diagonalizes the double evolution matrix. Then the second exclusive matrix $\overline{\mathcal{S}}$ is obtained from the relation

$$
\overline{\mathcal{S}}=\bar{T}^{-1} \mathcal{S} T^{-1} \mathcal{S}^{\dagger} \bar{T}^{-1}
$$


which is always correct for the Racah matrices [153] and follows from triviality of two unlinked unknots [29].

\subsection{Families of arborescent knots}

This approach to the exclusive Racah matrices is yet another impressive success of the evolution method $[87,144,145]$, which describes each knot or link together with a whole family which arises when any of the encountered $\mathcal{R}$-matrices is raised to an arbitrary power. The point is that calculations for the entire family is technically the same, but one obtains this way the HOMFLY polynomial for many knots at once, and also a new parameter, in which interesting recursions, of course, immediately arise. Most important, this provides a new ordering in the space of knots, which unifies knots of a similar complexity, which has nothing to do with the number of crossings used in the Rolfsen table. First examples of this family method application are provided in [30] and [32]. The actual tabulation of colored knot polynomials in [36], basing on [29]-[35] was made possible only by use of this method.

\subsection{Universal knot polynomials}

It is not easy to include conjugate representations, which will involve the rank $N$ dependence, within highest weight method which is $N$-independent. Interestingly for adjoint representations, Vogel's universality hypothesis [154] claims that they can be formulated in a universal, group-independent way. The hypothesis actually originated from knot theory studies, and the idea was to raise it up to the group theory level, where it partly failed. However, not very surprisingly, the knot polynomials are not sensitive to the failures, and they are indeed universal $[1,141]$. Moreover, an extension of Vogel's hypothesis from the dimensions and Casimirs to the Racah matrices, which is one of the steps required for evaluating the adjoint HOMFLY polynomial, also provided the non-trivial confirmation of the eigenvalue hypothesis and explicit formulas for the $6 \times 6$ Racah matrices [1]. This data has been useful for writing colored HOMFLY and colored Kauffman for adjoint representation.

\subsection{A collection of colored knot polynomials}

As we cited throughout this paper, the data on the colored knot polynomials are collected in the website [36]. We briefly describe here the structure of this site.

Basically, it consists of three large parts (apart from links to other knot tables that contain only uncolored knot polynomials with a notable exception of the colored Jones ones): the first part contains a description of some important families of knots and links; the second one contains a theoretical part with links to papers useful for evaluation of colored polynomials; and, the most important third part contains the data: the Racah and mixing matrices, which allow one to evaluate knot polynomials, and the results of this evaluation.

The results are: (i) for the HOMFLY polynomials [119], (ii) for the Kauffman polynomials [120] and (iii) for the universal polynomials [121]. Most of the results are either for knots from the Rolfsen table or for special families of knots. Since the case of HOMFLY 
polynomials is most developed, section (i) contains, apart from the polynomials themselves some additional information about the structure of the answers mostly related with their differential expansion. At last, this section contains the LMOV integers up to the fourth level for all arborescent knots from the Rolfsen table with no more than 8 crossings which have a 3 -strand braid representation. These are the knots: $3_{1}, 4_{1}, 5_{1}, 5_{2}, 6_{2}, 6_{3}, 7_{1}, 7_{3}, 7_{5}$, $8_{2}, 8_{5}, 8_{7}, 8_{9}, 8_{10}, 8_{16}, 8_{17}, 8_{18}, 8_{19}, 8_{20}, 8_{21}$. Similarly, section (ii), which has been less developed yet contains, apart from the Kauffman polynomials only the LMOV integers up to level two for the same set of knots. These LMOV tables are exactly the data obtained as a result of the present paper.

\section{Tests of integrality conjectures}

\section{1 $\mathrm{SU}(N)$ Chern-Simons}

As discussed in the introduction, the integrality conjecture (2.5) famously known as LMOV condition has been proven in $[122,123]$. Our focus in this paper is to write integer coefficients for the representations $Q$ with $|Q| \leq 4$. The HOMFLY polynomials for these representations and a list of the integers for more knots from the Rolfsen table [131] can be found in [36]. Here we present for a knot $8_{20}$ from the Rolfsen table. This knot $8_{20}$ is an arborescent and can also be obtained from 3-strand braid. The reason for our choice is that the exclusive Racah matrices necessary for evaluating the arborescent knots [32] are yet unavailable for the representation [3,1] [35], while the inclusive Racah matrices in this representation are known [33]. Hence, the integers in representations up to the fourth level can be constructed only for the knots that have 3 -strand braid representations. The answers for these integers are summarized in the tables below.

Note that one may think the integrality of these numbers trivially follows from the integrality of the HOMFLY coefficients. In fact, this is completely non-trivial: if one considers just the HOMFLY polynomials rescaled with the framing factor $(-1)^{p|R|} q^{2 p \varphi_{R}([2])}$, we see that these framed HOMFLY polynomials also obeys the integrality property. For example, one of the $p$ dependent coefficient, with this factor multiplied $A^{-2}\left(q-q^{-1}\right)^{9}$, is

$$
\begin{aligned}
N_{[2],-2,6}= & \frac{1}{3832012800}\left(148 p^{12}-2736 p^{11}+79112 p^{10}-831600 p^{9}+10539474 p^{8}-68756688 p^{7}+436908296 p^{6}\right. \\
& -1721451600 p^{5}+5409488128 p^{4}-11272637376 p^{3}+15223732992 p^{2}+2338875 \cdot(-1)^{p} \\
& -11844403200 p+3829673925) .
\end{aligned}
$$

Note that the huge denominator $3832012800=12 ! 2^{3}$ gets cancelled with the numerator for integer values of $p$. Further we observe that the number of non-zero integers increases with increasing $|p|$ starting from large enough values of $p$, and the integers themselves celebrate some additional constraints, e.g.

$$
N_{[p]}(p)=(-1)^{p} N_{\left[1^{p}\right]}(p+1) .
$$

Looking at the tables below, one may note the two properties: all the numbers in each column have the same sign (it alternates with turning at some value) and the sum of all the 
coefficients in each row is equal to zero. The first property, though being correct very often still sometimes breaks: for instance, for the twist knots (which have maximal braid number at the given number of crossings) starting from knot $6_{1}$ already for Young diagrams of level 2. The second property follows from the fact that the unreduced HOMFLY polynomials are cancelled at $A=1$, and, hence, so do $f_{R}(2.5)$. From this latter formula and the fact that $\psi_{R}(\Delta)$ are symmetric group characters and, hence, are linearly independent it follows that

$$
\sum_{n} N_{\mathbf{Q}, n, k}=0
$$

at least, up to the level $|Q|=4$, where ${ }^{*} p_{\Delta}$ are all independent.

\section{Knot $8_{20}$.}

\begin{tabular}{|c|c|c|c|c|c|}
\hline \multirow{4}{*}{$\mathbf{N}_{[1]}:$} & $k \backslash n=$ & -5 & -3 & -1 & 1 \\
\hline & 0 & 2 & -6 & 5 & -1 \\
\hline & 1 & 1 & -5 & 5 & -1 \\
\hline & 2 & 0 & -1 & 1 & 0 \\
\hline
\end{tabular}

$\mathbf{N}_{[2]}:$\begin{tabular}{|c|ccccccc|}
\hline$k \backslash n=$ & -10 & -8 & -6 & -4 & -2 & 0 & 2 \\
\hline 0 & 16 & -73 & 131 & -114 & 46 & -5 & -1 \\
1 & 50 & -231 & 400 & -319 & 111 & -10 & -1 \\
2 & 63 & -309 & 521 & -373 & 104 & -6 & 0 \\
3 & 37 & -212 & 359 & -231 & 48 & -1 & 0 \\
4 & 10 & -77 & 135 & -79 & 11 & 0 & 0 \\
5 & 1 & -14 & 26 & -14 & 1 & 0 & 0 \\
6 & 0 & -1 & 2 & -1 & 0 & 0 & 0 \\
\hline
\end{tabular}

$\mathbf{N}_{[1,1]}:$\begin{tabular}{|c|cccccc|}
\hline$k \backslash n=$ & -10 & -8 & -6 & -4 & -2 & 0 \\
\hline 0 & 25 & -115 & 210 & -190 & 85 & -15 \\
1 & 95 & -440 & 775 & -645 & 250 & -35 \\
2 & 155 & -743 & 1267 & -953 & 302 & -28 \\
3 & 129 & -680 & 1148 & -781 & 193 & -9 \\
4 & 56 & -354 & 607 & -377 & 69 & -1 \\
5 & 12 & -104 & 185 & -106 & 13 & 0 \\
6 & 1 & -16 & 30 & -16 & 1 & 0 \\
7 & 0 & -1 & 2 & -1 & 0 & 0 \\
\hline
\end{tabular}

$\mathbf{N}_{[3]}: \quad: \quad$\begin{tabular}{cccccccccccc|}
\hline$k \backslash n=$ & -15 & -13 & -11 & -9 & -7 & -5 & -3 & -1 & 1 & 3 \\
\hline 0 & 352 & -2125 & 5468 & -7791 & 6673 & -3470 & 1022 & -111 & -27 & 9 \\
1 & 3256 & -18695 & 44944 & -58584 & 44782 & -20245 & 5097 & -473 & -123 & 41 \\
2 & 14770 & -81370 & 183559 & -218724 & 147871 & -56664 & 11559 & -853 & -209 & 61 \\
3 & 41511 & -222579 & 475465 & -520438 & 310866 & -99510 & 15655 & -842 & -165 & 37 \\
4 & 77904 & -414115 & 847003 & -857240 & 453256 & -120239 & 13971 & -484 & -66 & 10 \\
5 & 101052 & -543578 & 1076296 & -1013191 & 474743 & -103702 & 8552 & -160 & -13 & 1 \\
6 & 92372 & -514010 & 995015 & -874977 & 362651 & -64643 & 3621 & -28 & -1 & 0 \\
7 & 60098 & -354425 & 676451 & -557025 & 202945 & -29084 & 1042 & -2 & 0 & 0 \\
8 & 27855 & -179063 & 339359 & -261883 & 82860 & -9322 & 194 & 0 & 0 & 0 \\
9 & 9107 & -66077 & 125094 & -90413 & 24338 & -2070 & 21 & 0 & 0 & 0 \\
10 & 2048 & -17576 & 33405 & -22574 & 4998 & -302 & 1 & 0 & 0 & 0 \\
11 & 301 & -3277 & 6279 & -3957 & 680 & -26 & 0 & 0 & 0 & 0 \\
12 & 26 & -406 & 787 & -461 & 55 & -1 & 0 & 0 & 0 & 0 \\
13 & 1 & -30 & 59 & -32 & 2 & 0 & 0 & 0 & 0 & 0 \\
14 & 0 & -1 & 2 & -1 & 0 & 0 & 0 & 0 & 0 & 0 \\
\hline
\end{tabular}




\begin{tabular}{|c|cccccccccc|}
\hline$k \backslash n=$ & -15 & -13 & -11 & -9 & -7 & -5 & -3 & -1 & 1 & 3 \\
\hline 0 & 1096 & -6812 & 18055 & -26511 & 23427 & -12647 & 4021 & -661 & 25 & 7 \\
1 & 11740 & -69190 & 171467 & -231418 & 183976 & -86971 & 23475 & -3220 & 118 & 23 \\
2 & 62734 & -352952 & 820140 & -1016046 & 721600 & -293594 & 64694 & -6802 & 204 & 22 \\
3 & 211059 & -1148176 & 2521968 & -2880042 & 1826520 & -634085 & 110761 & -8177 & 164 & 8 \\
4 & 482979 & -2581587 & 5415098 & -5735572 & 3255233 & -960113 & 130062 & -6167 & 66 & 1 \\
5 & 780717 & -4170723 & 8440154 & -8341780 & 4245634 & -1060226 & 109232 & -3021 & 13 & 0 \\
6 & 912409 & -4956911 & 9766956 & -9052011 & 4133483 & -869612 & 66643 & -958 & 1 & 0 \\
7 & 782632 & -4402394 & 8512833 & -7424291 & 3035652 & -533774 & 29531 & -189 & 0 & 0 \\
8 & 496928 & -2950758 & 5637051 & -4636024 & 1688623 & -245177 & 9378 & -21 & 0 & 0 \\
9 & 234028 & -1499349 & 2845722 & -2209021 & 710203 & -83655 & 2073 & -1 & 0 & 0 \\
10 & 81306 & -576851 & 1092898 & -800716 & 223914 & -20853 & 302 & 0 & 0 & 0 \\
11 & 20526 & -166688 & 316505 & -218681 & 51995 & -3683 & 26 & 0 & 0 & 0 \\
12 & 3656 & -35556 & 67891 & -44172 & 8616 & -436 & 1 & 0 & 0 & 0 \\
13 & 435 & -5426 & 10448 & -6389 & 963 & -31 & 0 & 0 & 0 & 0 \\
14 & 31 & -560 & 1090 & -625 & 65 & -1 & 0 & 0 & 0 & 0 \\
15 & 1 & -35 & 69 & -37 & 2 & 0 & 0 & 0 & 0 & 0 \\
16 & 0 & -1 & 2 & -1 & 0 & 0 & 0 & 0 & 0 & 0 \\
\hline
\end{tabular}




\begin{tabular}{|c|ccccccccc|}
\hline$k \backslash n=$ & -15 & -13 & -11 & -9 & -7 & -5 & -3 & -1 & 1 \\
\hline 0 & 817 & -5202 & 14122 & -21247 & 19265 & -10712 & 3552 & -647 & 52 \\
1 & 9896 & -59591 & 151295 & -209830 & 172040 & -84226 & 23656 & -3445 & 205 \\
2 & 60278 & -345235 & 821309 & -1048249 & 772553 & -328797 & 76150 & -8309 & 300 \\
3 & 232831 & -1283378 & 2881690 & -3396607 & 2249185 & -827003 & 155091 & -12020 & 211 \\
4 & 616432 & -3318766 & 7100687 & -7772468 & 4632525 & -1466503 & 219517 & -11501 & 77 \\
5 & 1162736 & -6209959 & 12783754 & -13070245 & 7025091 & -1908577 & 224707 & -7521 & 14 \\
6 & 1600769 & -8613912 & 17213476 & -16520487 & 8014837 & -1860533 & 169213 & -3364 & 1 \\
7 & 1634944 & -9004917 & 17603582 & -15919558 & 6965468 & -1372676 & 94164 & -1007 & 0 \\
8 & 1251705 & -7174091 & 13810549 & -11798458 & 4641249 & -769287 & 38525 & -192 & 0 \\
9 & 721849 & -4384030 & 8357680 & -6754633 & 2374468 & -326722 & 11409 & -21 & 0 \\
10 & 313286 & -2058624 & 3905215 & -2987163 & 929118 & -104204 & 2373 & -1 & 0 \\
11 & 101531 & -740262 & 1403124 & -1015440 & 275229 & -24510 & 328 & 0 & 0 \\
12 & 24156 & -201838 & 383609 & -262392 & 60556 & -4118 & 27 & 0 & 0 \\
13 & 4090 & -40952 & 78280 & -50529 & 9577 & -467 & 1 & 0 & 0 \\
14 & 466 & -5985 & 11536 & -7013 & 1028 & -32 & 0 & 0 & 0 \\
15 & 32 & -595 & 1159 & -662 & 67 & -1 & 0 & 0 & 0 \\
16 & 1 & -36 & 71 & -38 & 2 & 0 & 0 & 0 & 0 \\
17 & 0 & -1 & 2 & -1 & 0 & 0 & 0 & 0 & 0 \\
\hline
\end{tabular}




\begin{tabular}{|c|c|c|c|c|c|c|c|c|c|c|c|c|c|c|}
\hline & $k \backslash n=$ & -20 & -18 & -16 & -14 & -12 & -10 & -8 & -6 & -4 & -2 & 0 & 2 & 4 \\
\hline & 0 & 11440 & -87173 & 293893 & -576270 & 726572 & -614639 & 352840 & -135087 & 31946 & -3116 & -645 & 269 & -30 \\
\hline & 1 & 228250 & -1635276 & 5137191 & -9286702 & 10657519 & -8081601 & 4086664 & -1355188 & 275713 & -23835 & -4413 & 1934 & -256 \\
\hline & 2 & 2386083 & -16136564 & 47369508 & -79062965 & 82549940 & -55906878 & 24662653 & -6936301 & 1167700 & -85260 & -13024 & 6054 & -946 \\
\hline & 3 & 16661172 & -107057436 & 295234625 & -456862001 & 435207944 & -263432562 & 101033685 & -23791571 & 3205258 & -186183 & -21779 & 10693 & -1845 \\
\hline & 4 & 84507887 & -519907050 & 1355251916 & -1954018736 & 1704701161 & -924387333 & 308130762 & -60328166 & 6336339 & -273569 & -22825 & 11672 & -2058 \\
\hline & 5 & 324218115 & -1925277128 & 4773608162 & -6443894762 & 5167017105 & -2515811031 & 729145727 & -118202574 & 9488612 & -283394 & -15660 & 8205 & -1377 \\
\hline & 6 & 964060168 & -5570786966 & 13216800689 & -16777389869 & 12401705555 & -5430400831 & 1368154785 & -182973872 & 11046274 & -212013 & -7130 & 3771 & -561 \\
\hline & 7 & 2260162822 & -12811019508 & 29245594272 & -35043455568 & 23932680078 & -9429839738 & 2062360089 & -226492258 & 10126690 & -115736 & -2131 & 1124 & -136 \\
\hline & 8 & 4233247530 & -23722411643 & 52372794915 & -59428427145 & 37551473309 & -13307457043 & 2519220675 & -225742273 & 7347950 & -46065 & -401 & 209 & -18 \\
\hline & 9 & 6401577363 & -35742184785 & 76665320902 & -82599999575 & 48322505041 & -15378284326 & 2508707131 & -181847621 & 4219095 & -13203 & -43 & 22 & -1 \\
\hline \multirow{17}{*}{$\mathbf{N}_{[4]}:$} & 10 & 7882325987 & -44189952927 & 92475676765 & -94805633387 & 51345839966 & -14636437277 & 2044843043 & -118567832 & 1908313 & -2650 & -2 & 1 & 0 \\
\hline & 11 & 7955332198 & -45135236322 & 92502848394 & -90392118364 & 45284233622 & -11520499158 & 1367294612 & -62528465 & 673836 & -353 & 0 & 0 & 0 \\
\hline & 12 & 6613694775 & -38282967105 & 77104986495 & -71912996672 & 33272091742 & -7518694886 & 750290984 & -26588381 & 183076 & -28 & 0 & 0 & 0 \\
\hline & 13 & 4544009056 & -27063745212 & 53736998578 & -47883973931 & 20411212868 & -4072813151 & 337335831 & -9061459 & 37421 & -1 & 0 & 0 & 0 \\
\hline & 14 & 2584289462 & -15981116400 & 31372940707 & -26729420170 & 10461927572 & -1829952056 & 123776261 & -2450930 & 5554 & 0 & 0 & 0 & 0 \\
\hline & 15 & 1216390788 & -7887415145 & 15349485952 & -12510461549 & 4475947275 & -680239806 & 36810141 & -518220 & 564 & 0 & 0 & 0 & 0 \\
\hline & 16 & 472819775 & -3250240468 & 6285479701 & -4902402599 & 1593840148 & -208191240 & 8778345 & -83697 & 35 & 0 & 0 & 0 & 0 \\
\hline & 17 & 151097941 & -1114995248 & 2147461941 & -1603116813 & 469971253 & -52061194 & 1652078 & -9959 & 1 & 0 & 0 & 0 & 0 \\
\hline & 18 & 39405913 & -316778506 & 608869085 & -435066095 & 113847320 & -10516465 & 239570 & -822 & 0 & 0 & 0 & 0 & 0 \\
\hline & 19 & 8294017 & -73942242 & 142098694 & -97183015 & 22394545 & -1687755 & 25798 & -42 & 0 & 0 & 0 & 0 & 0 \\
\hline & 20 & 1385933 & -14015257 & 26975820 & -17655709 & 3517260 & -209987 & 1941 & -1 & 0 & 0 & 0 & 0 & 0 \\
\hline & 21 & 179446 & -2120970 & 4095152 & -2564506 & 430297 & -19510 & 91 & 0 & 0 & 0 & 0 & 0 & 0 \\
\hline & 22 & 17344 & -249997 & 484911 & -290476 & 39489 & -1273 & 2 & 0 & 0 & 0 & 0 & 0 & 0 \\
\hline & 23 & 1177 & -22102 & 43125 & -24704 & 2556 & -52 & 0 & 0 & 0 & 0 & 0 & 0 & 0 \\
\hline & 24 & 50 & -1378 & 2708 & -1483 & 104 & -1 & 0 & 0 & 0 & 0 & 0 & 0 & 0 \\
\hline & 25 & 1 & -54 & 107 & -56 & 2 & 0 & 0 & 0 & 0 & 0 & 0 & 0 & 0 \\
\hline & 26 & 0 & -1 & 2 & -1 & 0 & 0 & 0 & 0 & 0 & 0 & 0 & 0 & 0 \\
\hline
\end{tabular}




\begin{tabular}{|c|c|c|c|c|c|c|c|c|c|c|c|c|c|c|}
\hline & $k \backslash n=$ & -20 & -18 & -16 & -14 & -12 & -10 & -8 & -6 & -4 & -2 & 0 & 2 & 4 \\
\hline & 0 & 45142 & -356604 & 1248004 & -2544260 & 3341462 & -2951474 & 1776512 & -721950 & 191238 & -30194 & 2020 & 130 & -26 \\
\hline & 1 & 1061746 & -7886896 & 25774891 & -48658404 & 58570857 & -46814370 & 25091891 & -8900632 & 2010383 & -265010 & 14986 & 704 & -146 \\
\hline & 2 & 13219093 & -92644735 & 283364735 & -495881207 & 546856462 & -394544930 & 187217848 & -57276214 & 10767963 & -1129629 & 49369 & 1575 & -330 \\
\hline & 3 & 110978071 & -738218369 & 2123395303 & -3457325739 & 3501914639 & -2283015929 & 957875396 & -251314127 & 38722459 & -3108818 & 95608 & 1880 & -374 \\
\hline & 4 & 683626377 & -4346342959 & 11823796029 & -17993793212 & 16798542200 & -9918676313 & 3682017558 & -826512903 & 103384154 & -6163505 & 121495 & 1310 & -231 \\
\hline & 5 & 3220746083 & -19713056578 & 51012920266 & -72893664160 & 62941377502 & -33738218362 & 11091817656 & -2127319330 & 214560357 & -9270796 & 106893 & 548 & -79 \\
\hline & 6 & 11902797889 & -70635231409 & 174837831840 & -235577769444 & 188722760807 & -92013045366 & 26804397519 & -4385326880 & 354369716 & -10851594 & 66801 & 135 & -14 \\
\hline & 7 & 35131751461 & -203521505109 & 484315599846 & -617676693690 & 460269219324 & -204383089677 & 52746115436 & -7343388151 & 471962933 & -10002267 & 29877 & 18 & -1 \\
\hline & 8 & 83977371481 & -478017884947 & 1098713484889 & -1330695425149 & 924190522239 & -374008482052 & 85421742814 & -10084961299 & 510913581 & -7291050 & 9492 & 1 & 0 \\
\hline & 9 & 164416998734 & -925383009601 & 2063046363926 & -2379476149635 & 1542491103249 & -568872395551 & 114766633622 & -11437048121 & 451701955 & -4200668 & 2090 & 0 & 0 \\
\hline & 10 & 266136440145 & -1490091389662 & 3234417542766 & -3560978652096 & 2156541392942 & -724294109728 & 128707348520 & -10763632855 & 326963860 & -1904195 & 303 & 0 & 0 \\
\hline & 11 & 358904242033 & -2011015976626 & 4264679863987 & -4490663780569 & 2541586916172 & -776296340555 & 121046412016 & -8434521195 & 193857944 & -673233 & 26 & 0 & 0 \\
\hline & 12 & 405783962986 & -2288990129732 & 4757265150324 & -4798780206020 & 2537845729254 & -703490616645 & 95785780542 & -5513478795 & 93991109 & -183024 & 1 & 0 & 0 \\
\hline & 13 & 386560168725 & -2208448533446 & 4511053037295 & -4364839402142 & 2155514108908 & -540773914262 & 63904984526 & -3007528055 & 37115870 & -37419 & 0 & 0 & 0 \\
\hline \multirow{17}{*}{$\mathbf{N}_{[2,2]}$} & 14 & 311445592580 & -1813201702273 & 3649582846272 & -3390820641320 & 1561745471082 & -353369057566 & 35972944947 & -1367302839 & 11854671 & -5554 & 0 & 0 & 0 \\
\hline & 15 & 212773261251 & -1270437768319 & 2525765933165 & -2255190702268 & 967019262973 & -196491288944 & 17074742058 & -516469165 & 3029813 & -564 & 0 & 0 & 0 \\
\hline & 16 & 123435129401 & -760994699619 & 1497674084990 & -1285908719153 & 512097029310 & -92961530399 & 6819326161 & -161230450 & 609794 & -35 & 0 & 0 & 0 \\
\hline & 17 & 60818637949 & -389973798110 & 761283513563 & -628846628374 & 231845177029 & -37368435345 & 2282702661 & -41263734 & 94362 & -1 & 0 & 0 & 0 \\
\hline & 18 & 25423341446 & -170894213960 & 331532675036 & -263555527248 & 89592696958 & -12727026265 & 636599926 & -8556713 & 10820 & 0 & 0 & 0 & 0 \\
\hline & 19 & 8993174943 & -63934192335 & 123473129260 & -94483488337 & 29462284035 & -3656119893 & 146625003 & -1413541 & 865 & 0 & 0 & 0 & 0 \\
\hline & 20 & 2680538218 & -20357277502 & 39200955717 & -28877797256 & 8206333118 & -880120523 & 27549660 & -181475 & 43 & 0 & 0 & 0 & 0 \\
\hline & 21 & 668955054 & -5490782054 & 10558350417 & -7487841536 & 1923099476 & -175913501 & 4149580 & -17437 & 1 & 0 & 0 & 0 & 0 \\
\hline & 22 & 138520892 & -1246055050 & 2395991231 & -1635750854 & 375629940 & -28823523 & 488543 & -1179 & 0 & 0 & 0 & 0 & 0 \\
\hline & 23 & 23501434 & -235692966 & 453774067 & -298191597 & 60368864 & -3803029 & 43277 & -50 & 0 & 0 & 0 & 0 & 0 \\
\hline & 24 & 3209465 & -36680505 & 70793497 & -44771764 & 7840441 & -393844 & 2711 & -1 & 0 & 0 & 0 & 0 & 0 \\
\hline & 25 & 343919 & -4612864 & 8934653 & -5436982 & 801973 & -30806 & 107 & 0 & 0 & 0 & 0 & 0 & 0 \\
\hline & 26 & 27830 & -456835 & 888927 & -520375 & 62161 & -1710 & 2 & 0 & 0 & 0 & 0 & 0 & 0 \\
\hline & 27 & 1598 & -34280 & 67076 & -37764 & 3430 & -60 & 0 & 0 & 0 & 0 & 0 & 0 & 0 \\
\hline & 28 & 58 & -1831 & 3606 & -1952 & 120 & -1 & 0 & 0 & 0 & 0 & 0 & 0 & 0 \\
\hline & 29 & 1 & -62 & 123 & -64 & 2 & 0 & 0 & 0 & 0 & 0 & 0 & 0 & 0 \\
\hline & 30 & 0 & -1 & 2 & -1 & 0 & 0 & 0 & 0 & 0 & 0 & 0 & 0 & 0 \\
\hline
\end{tabular}




\begin{tabular}{|c|c|c|c|c|c|c|c|c|c|c|c|c|c|}
\hline$k \backslash n=$ & -20 & -18 & -16 & -14 & -12 & -10 & -8 & -6 & -4 & -2 & 0 & 2 & 4 \\
\hline 0 & 54046 & -421913 & 1458902 & -2937624 & 3808680 & -3318671 & 1968098 & -785583 & 202064 & -29280 & 920 & 415 & -54 \\
\hline 1 & 1207930 & -8866816 & 28610436 & -53272255 & 63169584 & -49668944 & 26149099 & -9092365 & 2000388 & -246716 & 7507 & 2500 & -348 \\
\hline 2 & 14241824 & -98651137 & 297765825 & -513306085 & 556454090 & -393694624 & 182719619 & -54537515 & 9968959 & -992881 & 26593 & 6264 & -932 \\
\hline 3 & 112850652 & -742218311 & 2106166482 & -3374445171 & 3353073386 & -2136652475 & 872510806 & -221807292 & 32987994 & -2526777 & 53538 & 8456 & -1288 \\
\hline 4 & 653965466 & -4113310890 & 11037788852 & -16513730121 & 15095228135 & -8683571028 & 3121017399 & -673245823 & 80317835 & -4533288 & 67664 & 6800 & -1001 \\
\hline 5 & 2888733279 & -17506113346 & 44687098358 & -62724026193 & 52933567411 & -27554780281 & 8724052977 & -1593494579 & 150952041 & -6049005 & 56385 & 3408 & -455 \\
\hline 6 & 9975276084 & -58675127063 & 143285992377 & -189504490234 & 148106635013 & -69899466805 & 19503109153 & -3010288126 & 224500982 & -6174069 & 31735 & 1073 & -120 \\
\hline 7 & 27413969447 & -157632856262 & 370192623268 & -463092032177 & 336031568249 & -143958430444 & 35384632338 & -4602450447 & 267852157 & -4888444 & 12126 & 206 & -17 \\
\hline 8 & 60794764793 & -344088847169 & 780828884205 & -926938058004 & 625678990120 & -243423323913 & 52639550626 & -5747305587 & 258360292 & -3018483 & 3099 & 22 & -1 \\
\hline 9 & 110022802044 & -617025495856 & 1358843153531 & -1535082506469 & 965128511825 & -340890212578 & 64701897506 & -5898989939 & 202291605 & -1452177 & 507 & 1 & 0 \\
\hline 10 & 163995554522 & -917250958025 & 1968031062413 & -2120675962227 & 1242763450545 & -398084069590 & 66090861594 & -4998150672 & 128752218 & -540826 & 48 & 0 & 0 \\
\hline 11 & 202863894726 & -1138898560506 & 2389144523104 & -2460370558556 & 1344113111105 & -389755815577 & 56342255075 & -3505241905 & 66546492 & -153960 & 2 & 0 & 0 \\
\hline 12 & 209533734845 & -1188374089005 & 2445223534552 & -2410319996901 & 1227007686986 & -321262829015 & 40201070394 & -2036913022 & 27833969 & -32803 & 0 & 0 & 0 \\
\hline 13 & 181570438382 & -1047138101394 & 2119566035105 & -2002402805737 & 948918733102 & -223586809674 & 24043357815 & -980203313 & 9360768 & -5054 & 0 & 0 & 0 \\
\hline 14 & 132459443162 & -782044704685 & 1561389673170 & -1415147162422 & 623288956096 & -131612894137 & 12053834213 & -389650341 & 2505475 & -531 & 0 & 0 & 0 \\
\hline 15 & 81530208844 & -496280477552 & 979725736282 & -852553990370 & 348206309830 & -65558758888 & 5057797190 & -127350800 & 525498 & -34 & 0 & 0 & 0 \\
\hline 16 & 42377740331 & -267964462462 & 524233827595 & -438257130491 & 165484567211 & -27610978919 & 1770317014 & -33964645 & 84367 & -1 & 0 & 0 & 0 \\
\hline 17 & 18591995559 & -123124330894 & 239197147867 & -192192614652 & 66830581769 & -9809519063 & 514038540 & -7309123 & 9997 & 0 & 0 & 0 & 0 \\
\hline 18 & 6870751800 & -48086661572 & 92944396253 & -71797978690 & 22875962548 & -2928023746 & 122800998 & -1248414 & 823 & 0 & 0 & 0 & 0 \\
\hline 19 & 2130717160 & -15921473005 & 30670831701 & -22782692662 & 6608716033 & -729783904 & 23849804 & -165169 & 42 & 0 & 0 & 0 & 0 \\
\hline 20 & 551206991 & -4449819754 & 8557099836 & -6112760303 & 1601134345 & -150546606 & 3701797 & -16307 & 1 & 0 & 0 & 0 & 0 \\
\hline 21 & 117927509 & -1043083270 & 2005345733 & -1377645739 & 322395428 & -25386405 & 447874 & -1130 & 0 & 0 & 0 & 0 & 0 \\
\hline 22 & 20610727 & -203221777 & 391130409 & -258395591 & 53274001 & -3438391 & 40671 & -49 & 0 & 0 & 0 & 0 & 0 \\
\hline 23 & 2891884 & -32493291 & 62686783 & -39820710 & 7097419 & -364690 & 2606 & -1 & 0 & 0 & 0 & 0 & 0 \\
\hline 24 & 317631 & -4188592 & 8109422 & -4952537 & 743126 & -29155 & 105 & 0 & 0 & 0 & 0 & 0 & 0 \\
\hline 25 & 26289 & -424326 & 825338 & -484501 & 58849 & -1651 & 2 & 0 & 0 & 0 & 0 & 0 & 0 \\
\hline 26 & 1541 & -32510 & 63591 & -35875 & 3312 & -59 & 0 & 0 & 0 & 0 & 0 & 0 & 0 \\
\hline 27 & 57 & -1770 & 3485 & -1889 & 118 & -1 & 0 & 0 & 0 & 0 & 0 & 0 & 0 \\
\hline 28 & 1 & -61 & 121 & -63 & 2 & 0 & 0 & 0 & 0 & 0 & 0 & 0 & 0 \\
\hline 29 & 0 & -1 & 2 & -1 & 0 & 0 & 0 & 0 & 0 & 0 & 0 & 0 & 0 \\
\hline
\end{tabular}




\begin{tabular}{|c|c|c|c|c|c|c|c|c|c|c|c|c|c|c|}
\hline & $k \backslash n=$ & -20 & -18 & -16 & -14 & -12 & -10 & -8 & -6 & -4 & -2 & 0 & 2 & 4 \\
\hline & 0 & 82226 & -655421 & 2315601 & -4768098 & 6328599 & -5653333 & 3444750 & -1419723 & 383294 & -62978 & 5093 & 17 & -27 \\
\hline & 1 & 2028650 & -15204532 & 50179191 & -95758205 & 116651501 & -94485833 & 51405282 & -18548132 & 4277813 & -584321 & 38779 & -69 & -124 \\
\hline & 2 & 26498440 & -187350248 & 578823419 & -1024679559 & 1145145541 & -839062818 & 405427370 & -126727185 & 24457479 & -2665562 & 133830 & -498 & -209 \\
\hline & 3 & 233347245 & -1565447193 & 4548843934 & -7496837137 & 7704425860 & -5111284629 & 2190726176 & -590195832 & 94065559 & -7920853 & 277991 & -956 & -165 \\
\hline & 4 & 1507954814 & -9664436055 & 26558995186 & -40929741986 & 38809049674 & -23361113876 & 8885693743 & -2058362496 & 268617640 & -17043660 & 387951 & -869 & -66 \\
\hline & 5 & 7457193738 & -45979882379 & 120177405182 & -173955538607 & 152695842572 & -83583875475 & 28235741941 & -5615477154 & 596110616 & -27903222 & 383230 & -429 & -13 \\
\hline & 6 & 28953907377 & -172943961905 & 432241801187 & -590126024957 & 481008054221 & -239881644844 & 72003333217 & -12273244597 & 1053142525 & -35636775 & 274670 & -118 & -1 \\
\hline & 7 & 89884104520 & -523567063468 & 1257592724079 & -1625517784143 & 1233501689839 & -561205891237 & 149658769719 & -21813116596 & 1502387244 & -35964061 & 144121 & -17 & 0 \\
\hline & 8 & 226269108658 & -1293486377314 & 2999627442970 & -3682825729662 & 2607163010053 & -1082986025538 & 256372018615 & -31850983340 & 1746335725 & -28855399 & 55233 & -1 & 0 \\
\hline & 9 & 467205567433 & -2637141435217 & 5929009498223 & -6934041621715 & 4586535710544 & -1739735704517 & 365019802865 & -38497118179 & 1663725933 & -18440624 & 15254 & 0 & 0 \\
\hline & 10 & 798830764924 & -4478406223815 & 9798267944342 & -10941736201568 & 6769351924270 & -2343670711258 & 434797827759 & -38729796224 & 1303841022 & -9372403 & 2951 & 0 & 0 \\
\hline & 11 & 1139985271543 & -6384240759439 & 13639312613495 & -14572599444620 & 8437189046119 & -2663427324899 & 435504330724 & -32561954543 & 841991174 & -3769933 & 379 & 0 & 0 \\
\hline & 12 & 1366722396147 & -7689612276847 & 16091251780551 & -16476684402749 & 8928116418760 & -2565505362470 & 368202683338 & -22938044558 & 447997301 & -1189502 & 29 & 0 & 0 \\
\hline & 13 & 1383894030779 & -7867197628677 & 16170858227109 & -15890895606776 & 8055469323002 & -2102225549823 & 263457376308 & -13555859647 & 195977951 & -290227 & 1 & 0 & 0 \\
\hline \multirow{18}{*}{$\mathbf{N}_{[2,1,1]}:$} & 14 & 1188437503303 & -6866008935580 & 13898411902941 & -13122154163682 & 6217461566062 & -1469268192825 & 159769985154 & -6719786231 & 70174408 & -53550 & 0 & 0 & 0 \\
\hline & 15 & 868235167489 & -5128234529678 & 10247154909485 & -9303838757754 & 4114596950171 & -877284165510 & 82139373931 & -2789359534 & 20418612 & -7212 & 0 & 0 & 0 \\
\hline & 16 & 540700909123 & -3285457404714 & 6494554360540 & -5674659729236 & 2337825783692 & -447764901222 & 35762403527 & -966195870 & 4774828 & -668 & 0 & 0 & 0 \\
\hline & 17 & 287299047689 & -1807791763055 & 3542327477812 & -2980255989623 & 1140801978628 & -195257317371 & 13153395604 & -277712529 & 882883 & -38 & 0 & 0 & 0 \\
\hline & 18 & 130217910487 & -854569778418 & 1662937142418 & -1347715231428 & 477747305355 & -72621490394 & 4069701441 & -65685459 & 125999 & -1 & 0 & 0 & 0 \\
\hline & 19 & 50272149115 & -346775317960 & 671281231115 & -524223299597 & 171370833030 & -22965602040 & 1052625131 & -12632167 & 13373 & 0 & 0 & 0 & 0 \\
\hline & 20 & 16483582606 & -120556205087 & 232518896575 & -175004119802 & 52480149044 & -6145934856 & 225572186 & -1941659 & 993 & 0 & 0 & 0 & 0 \\
\hline & 21 & 4569475871 & -35786540394 & 68870661235 & -49963676125 & 13653236118 & -1382474621 & 39550526 & -232656 & 46 & 0 & 0 & 0 & 0 \\
\hline & 22 & 1063907003 & -9025725427 & 17355193701 & -12136698507 & 2996715295 & -258945679 & 5574538 & -20925 & 1 & 0 & 0 & 0 & 0 \\
\hline & 23 & 206133310 & -1920640657 & 3694626649 & -2490504935 & 549639113 & -39867920 & 615768 & -1328 & 0 & 0 & 0 & 0 & 0 \\
\hline & 24 & 32812198 & -341545889 & 658045136 & -427554352 & 83147061 & -4955406 & 51305 & -53 & 0 & 0 & 0 & 0 & 0 \\
\hline & 25 & 4214933 & -50094771 & 96772118 & -60598209 & 10187511 & -484611 & 3030 & -1 & 0 & 0 & 0 & 0 & 0 \\
\hline & 26 & 425868 & -5950859 & 11537843 & -6962232 & 985144 & -35877 & 113 & 0 & 0 & 0 & 0 & 0 & 0 \\
\hline & 27 & 32567 & -557905 & 1086670 & -631778 & 72333 & -1889 & 2 & 0 & 0 & 0 & 0 & 0 & 0 \\
\hline & 28 & 1771 & -39712 & 77773 & -43557 & 3788 & -63 & 0 & 0 & 0 & 0 & 0 & 0 & 0 \\
\hline & 29 & 61 & -2016 & 3973 & -2143 & 126 & -1 & 0 & 0 & 0 & 0 & 0 & 0 & 0 \\
\hline & 30 & 1 & -65 & 129 & -67 & 2 & 0 & 0 & 0 & 0 & 0 & 0 & 0 & 0 \\
\hline & 31 & 0 & -1 & 2 & -1 & 0 & 0 & 0 & 0 & 0 & 0 & 0 & 0 & 0 \\
\hline
\end{tabular}




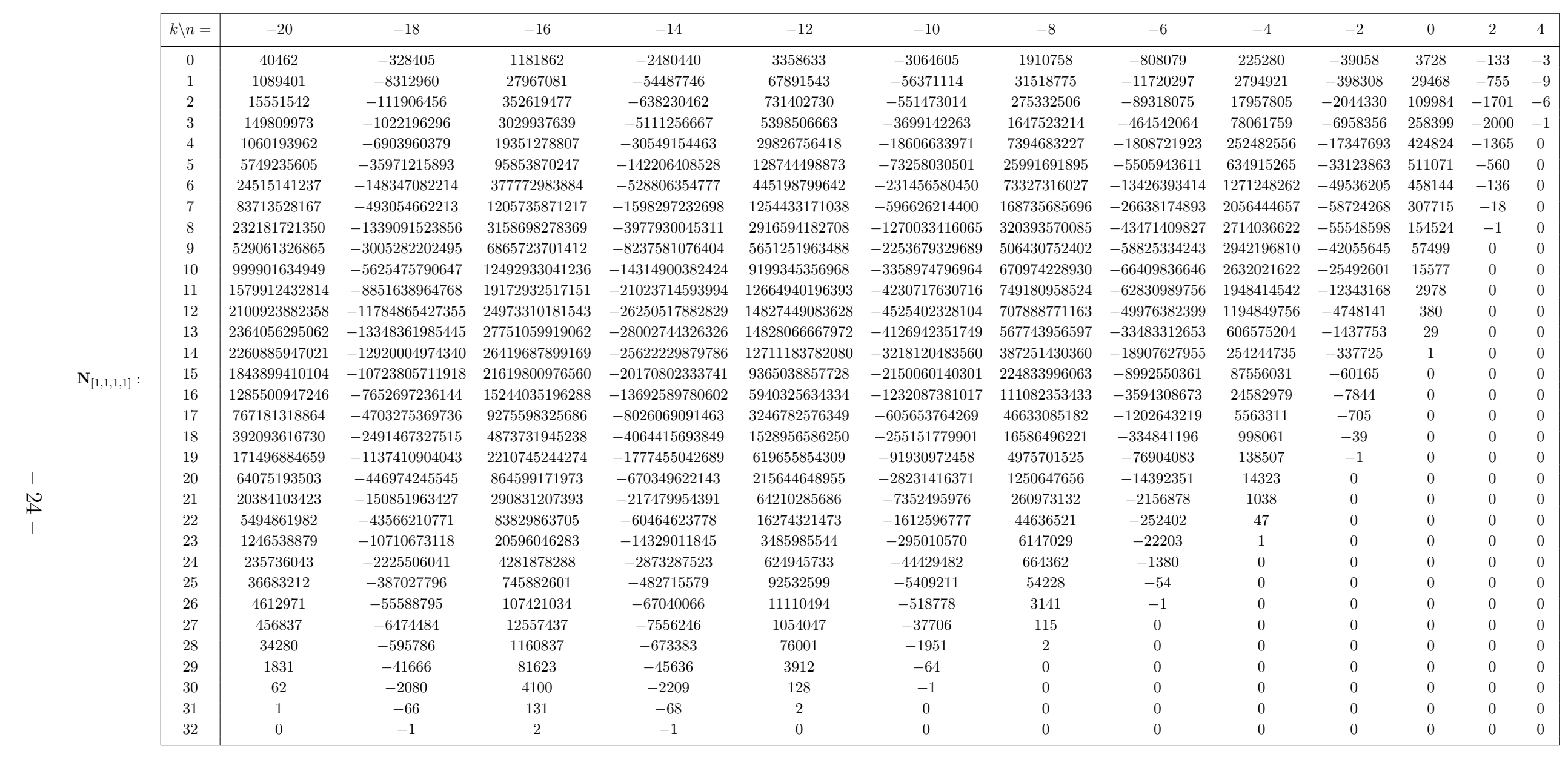




\subsection{SO/Sp Chern-Simons}

In this case, the integers (2.14) and (2.18) could be calculated for not so many representations as compared with the HOMFLY case, since the HOMFLY polynomials in composite representations are not available so far for exception of the adjoint representation. The answers for this latter one can be obtained for the Kauffman and HOMFLY cases at once from the universal adjoint knot polynomials, [1, 141]. Their explicit expressions, as well as the Kauffman polynomials in the fundamental representation and the HOMFLY polynomials in the fundamental, first symmetric and first antisymmetric representations, which are also necessary in this case, can be found in [36]. The universal adjoint knot polynomials have been constructed so far only for the arborescent knots. Since knot $8_{20}$ used as an example in the previous subsection is arborescent, we give the integers for this knot (the results for other arborescent knots can be again found in [36]). In fact, knot $8_{20}$ enjoys a peculiar property: $\hat{\mathbf{N}}_{[1]}^{c=2}=0$ for it. This property was conjectured in [125] for the torus knots, however, it turns out to be the case for some other knots too, though it is not met too often: in the Rolfsen table [131] only the knots $5_{2}, 7_{1}, 8_{20}, 9_{1}, 10_{125}, 10_{128}, 10_{132}, 10_{139}, 10_{161}$ and the torus knots $3_{1}, 5_{1}, 8_{19}, 10_{124}$ celebrate this property.

\section{Knot $8_{20}$.}

$\hat{\mathbf{N}}_{[1]}^{c=0}:$\begin{tabular}{c|cccc}
$k \backslash n=$ & -5 & -3 & -1 & 1 \\
\hline 0 & 4 & -12 & 10 & -2 \\
1 & 2 & -10 & 10 & -2 \\
2 & 0 & -2 & 2 & 0
\end{tabular}$\hat{\mathbf{N}}_{[1]}^{c=1}:$\begin{tabular}{ccccccc|}
$k \backslash n=$ & -6 & -4 & -2 & 0 & 2 \\
\hline 0 & 3 & -10 & 12 & -5 & 1 \\
1 & 4 & -15 & 16 & -5 & 0 \\
2 & 1 & -7 & 7 & -1 & 0 \\
3 & 0 & -1 & 1 & 0 & 0 \\
\hline
\end{tabular}

$\hat{\mathbf{N}}_{[2]}^{c=0}:=$\begin{tabular}{c|ccccccccc|}
\hline$k \backslash n=$ & -12 & -10 & -8 & -6 & -4 & -2 & 0 & 2 & 4 \\
\hline 0 & 9 & 22 & -216 & 462 & -442 & 194 & -25 & -6 & 1 \\
1 & 24 & 60 & -632 & 1340 & -1212 & 484 & -60 & -4 & 0 \\
2 & 22 & 84 & -817 & 1662 & -1364 & 458 & -45 & 0 & 0 \\
3 & 8 & 58 & -564 & 1106 & -804 & 208 & -12 & 0 & 0 \\
4 & 1 & 18 & -211 & 408 & -261 & 46 & -1 & 0 & 0 \\
5 & 0 & 2 & -40 & 78 & -44 & 4 & 0 & 0 & 0 \\
6 & 0 & 0 & -3 & 6 & -3 & 0 & 0 & 0 & 0 \\
\hline
\end{tabular}

$\hat{\mathbf{N}}_{[1,1]}^{c=0}:=$\begin{tabular}{|c|ccccccccc|}
\hline$k \backslash n=$ & -12 & -10 & -8 & -6 & -4 & -2 & 0 & 2 & 4 \\
\hline 0 & 9 & 40 & -300 & 620 & -594 & 272 & -45 & -4 & 1 \\
1 & 24 & 150 & -1050 & 2090 & -1864 & 762 & -110 & -2 & 0 \\
2 & 22 & 268 & -1685 & 3154 & -2524 & 854 & -89 & 0 & 0 \\
3 & 8 & 242 & -1500 & 2684 & -1904 & 498 & -28 & 0 & 0 \\
4 & 1 & 110 & -765 & 1352 & -857 & 162 & -3 & 0 & 0 \\
5 & 0 & 24 & -220 & 396 & -228 & 28 & 0 & 0 & 0 \\
6 & 0 & 2 & -33 & 62 & -33 & 2 & 0 & 0 & 0 \\
7 & 0 & 0 & -2 & 4 & -2 & 0 & 0 & 0 & 0 \\
\hline
\end{tabular}




\begin{tabular}{|c|cccccccc|}
\hline$k \backslash n=$ & -11 & -9 & -7 & -5 & -3 & -1 & 1 & 3 \\
\hline 0 & 163 & -723 & 1301 & -1217 & 631 & -179 & 25 & -1 \\
1 & 1459 & -6030 & 9636 & -7506 & 2970 & -589 & 61 & -1 \\
2 & 6463 & -25270 & 36235 & -23553 & 6911 & -836 & 50 & 0 \\
3 & 17319 & -66196 & 86737 & -47418 & 10208 & -667 & 17 & 0 \\
4 & 30172 & -116549 & 142053 & -65559 & 10204 & -323 & 2 & 0 \\
5 & 35400 & -142768 & 164366 & -63878 & 6974 & -94 & 0 & 0 \\
6 & 28479 & -123929 & 136524 & -44288 & 3229 & -15 & 0 & 0 \\
7 & 15809 & -76877 & 81912 & -21832 & 989 & -1 & 0 & 0 \\
8 & 6023 & -34068 & 35419 & -7565 & 191 & 0 & 0 & 0 \\
9 & 1542 & -10670 & 10902 & -1795 & 21 & 0 & 0 & 0 \\
10 & 253 & -2302 & 2325 & -277 & 1 & 0 & 0 & 0 \\
11 & 24 & -325 & 326 & -25 & 0 & 0 & 0 & 0 \\
12 & 1 & -27 & 27 & -1 & 0 & 0 & 0 & 0 \\
13 & 0 & -1 & 1 & 0 & 0 & 0 & 0 & 0 \\
\hline
\end{tabular}

N

$\hat{\mathbf{N}}_{[1,1]}^{c=1}: \quad$\begin{tabular}{c|cccccccc|}
\hline$k \backslash n=$ & -11 & -9 & -7 & -5 & -3 & -1 & 1 & 3 \\
\hline 0 & 208 & -944 & 1734 & -1642 & 842 & -222 & 24 & 0 \\
1 & 2107 & -8865 & 14524 & -11641 & 4676 & -856 & 55 & 0 \\
2 & 10561 & -41834 & 61612 & -41798 & 12948 & -1529 & 40 & 0 \\
3 & 32160 & -123599 & 166318 & -95920 & 22662 & -1632 & 11 & 0 \\
4 & 64264 & -247000 & 308745 & -151799 & 26894 & -1105 & 1 & 0 \\
5 & 87697 & -347013 & 408847 & -171220 & 22160 & -471 & 0 & 0 \\
6 & 83551 & -350248 & 393823 & -139736 & 12731 & -121 & 0 & 0 \\
7 & 56187 & -256993 & 278670 & -82900 & 5053 & -17 & 0 & 0 \\
8 & 26713 & -137658 & 145204 & -35610 & 1352 & -1 & 0 & 0 \\
9 & 8897 & -53635 & 55429 & -10923 & 232 & 0 & 0 & 0 \\
10 & 2026 & -14998 & 15275 & -2326 & 23 & 0 & 0 & 0 \\
11 & 300 & -2927 & 2952 & -326 & 1 & 0 & 0 & 0 \\
12 & 26 & -378 & 379 & -27 & 0 & 0 & 0 & 0 \\
13 & 1 & -29 & 29 & -1 & 0 & 0 & 0 & 0 \\
14 & 0 & -1 & 1 & 0 & 0 & 0 & 0 & 0 \\
\hline
\end{tabular}

$\hat{\mathbf{N}}_{[2]}^{c=2}:=$\begin{tabular}{|c|ccccccccc|}
\hline$k \backslash n=$ & -12 & -10 & -8 & -6 & -4 & -2 & 0 & 2 & 4 \\
\hline 0 & 248 & -954 & 1413 & -1015 & 399 & -138 & 65 & -21 & 3 \\
1 & 2419 & -9185 & 12990 & -8445 & 2575 & -469 & 155 & -41 & 1 \\
2 & 10970 & -40936 & 54318 & -31193 & 7435 & -708 & 143 & -29 & 0 \\
3 & 28819 & -107691 & 134330 & -67226 & 12320 & -606 & 63 & -9 & 0 \\
4 & 47840 & -183768 & 217177 & -93757 & 12806 & -310 & 13 & -1 & 0 \\
5 & 52677 & -213996 & 241766 & -89041 & 8686 & -93 & 1 & 0 & 0 \\
6 & 39561 & -175117 & 190771 & -59093 & 3893 & -15 & 0 & 0 & 0 \\
7 & 20519 & -102241 & 108245 & -27663 & 1141 & -1 & 0 & 0 & 0 \\
8 & 7335 & -42733 & 44274 & -9086 & 210 & 0 & 0 & 0 & 0 \\
9 & 1772 & -12673 & 12926 & -2047 & 22 & 0 & 0 & 0 & 0 \\
10 & 276 & -2601 & 2625 & -301 & 1 & 0 & 0 & 0 & 0 \\
11 & 25 & -351 & 352 & -26 & 0 & 0 & 0 & 0 & 0 \\
12 & 1 & -28 & 28 & -1 & 0 & 0 & 0 & 0 & 0 \\
13 & 0 & -1 & 1 & 0 & 0 & 0 & 0 & 0 & 0 \\
\hline
\end{tabular}

$\hat{\mathbf{N}}_{[1,1]}^{c=2}: \quad$\begin{tabular}{|c|cccccccccc|}
\hline$k \backslash n=$ & -12 & -10 & -8 & -6 & -4 & -2 & 0 & 2 & 4 \\
\hline 0 & 315 & -1214 & 1794 & -1260 & 438 & -100 & 36 & -10 & 1 \\
1 & 3465 & -13225 & 18885 & -12420 & 3714 & -470 & 66 & -15 & 0 \\
2 & 17878 & -67003 & 90314 & -53437 & 13273 & -1060 & 42 & -7 & 0 \\
3 & 53910 & -201207 & 255638 & -133587 & 26585 & -1349 & 11 & -1 & 0 \\
4 & 103753 & -394200 & 474873 & -216810 & 33397 & -1014 & 1 & 0 & 0 \\
5 & 134083 & -531391 & 611656 & -241661 & 27769 & -456 & 0 & 0 & 0 \\
6 & 120036 & -509029 & 564214 & -190755 & 15654 & -120 & 0 & 0 & 0 \\
7 & 75736 & -353077 & 379598 & -108244 & 6004 & -17 & 0 & 0 & 0 \\
8 & 33858 & -178831 & 187707 & -44274 & 1541 & -1 & 0 & 0 & 0 \\
9 & 10648 & -66054 & 68079 & -12926 & 253 & 0 & 0 & 0 & 0 \\
10 & 2301 & -17575 & 17875 & -2625 & 24 & 0 & 0 & 0 & 0 \\
11 & 325 & -3277 & 3303 & -352 & 1 & 0 & 0 & 0 & 0 \\
12 & 27 & -406 & 407 & -28 & 0 & 0 & 0 & 0 & 0 \\
13 & 1 & -30 & 30 & -1 & 0 & 0 & 0 & 0 & 0 \\
14 & 0 & -1 & 1 & 0 & 0 & 0 & 0 & 0 & 0 \\
\hline
\end{tabular}




\subsection{Link polynomials}

In the link case, already the lowest relations (2.22), (2.23) imply a non-trivial test: one has to check that the expansions (2.24) and (2.25), indeed, starts from 1 and $\left(q-q^{-1}\right)$ respectively, i.e. that $(2.23)$ cancels at $q=1$, while $(2.22)$ is regular. The literal integrality checks in this case require knowledge of a series of colored knot and link polynomials. We need to know: the HOMFLY and Kauffman polynomials for links and knots in the fundamental representation, which can be found in [131]; the HOMFLY polynomials of links when one of the link components is in the first (anti)symmetric representation, and the other one is in the fundamental one, which are calculated using the known exclusive Racah matrices [71, 72] or by the cabling method [80] and can be found in [36]; the HOMFLY polynomials of knots in the adjoint representation and the Kauffman polynomials of knots in the first (anti)symmetric representation, which are read off the universal knot polynomials [1] in [36]; the HOMFLY polynomials of links with one component in the adjoint representation and the other one in the fundamental one and similarly the Kauffman polynomials of links with one component in the first (anti)symmetric representation and the other one in the fundamental one, which can be constructed with the inclusive Racah matrices that we discuss in the next subsection (the manifest expressions for knot polynomials can be found in [36]). This finally allows us to obtain the integers, and we again write down them just for a link 7a3, while more examples can be found in [36]:

\begin{tabular}{|c|c|c|c|c|c|}
\hline \multirow{3}{*}{$\begin{array}{ll}1 & \hat{\mathbf{N}}_{[1],[1]}^{c=0} \\
\underset{\mathbf{N}}{\mathbf{N}} & =0\end{array}$} & $k \backslash n=$ & 0 & 2 & 4 & 6 \\
\hline & 0 & 8 & -24 & 24 & -8 \\
\hline & 1 & 4 & -20 & 20 & -4 \\
\hline & 2 & 0 & -4 & 4 & 0 \\
\hline
\end{tabular}

$\hat{\mathbf{N}}_{[1],[1]}^{c=1}:$\begin{tabular}{|c|ccccc|}
$k \backslash n=$ & 0 & 2 & 4 & 6 & 8 \\
\hline 0 & 0 & 0 & 0 & 0 & 0 \\
1 & 2 & -5 & 3 & 1 & -1 \\
2 & 1 & -5 & 4 & 0 & 0 \\
3 & 0 & -1 & 1 & 0 & 0 \\
\hline
\end{tabular}

$\hat{\mathbf{N}}_{[1],[1]}^{c=2}:$\begin{tabular}{|c|ccccc|}
\hline$k \backslash n=$ & 1 & 3 & 5 & 7 & 9 \\
\hline 0 & -5 & 14 & -12 & 2 & 1 \\
1 & -2 & 11 & -10 & 1 & 0 \\
2 & 0 & 2 & -2 & 0 & 0 \\
\hline
\end{tabular}

\begin{tabular}{|c|c|ccccc|}
$\hat{\mathbf{N}}_{[2],[1]}^{c=0}:$ & $k \backslash n=$ & -1 & 1 & 3 & 5 & 7 \\
\cline { 2 - 7 } & 0 & -4 & 24 & -48 & 40 & -12 \\
1 & -2 & 16 & -40 & 32 & -6 \\
2 & 0 & 2 & -8 & 6 & 0 \\
\hline
\end{tabular}

$\hat{\mathbf{N}}_{[2],[1]}^{c=1}:$\begin{tabular}{|c|cccc|}
\hline$k \backslash n=$ & 0 & 2 & 4 & 6 \\
\hline 0 & 0 & 0 & 0 & 0 \\
1 & 7 & -9 & 5 & -1 \\
2 & 6 & -9 & 5 & -1 \\
3 & 1 & -2 & 1 & 0 \\
\hline
\end{tabular}

$\hat{\mathbf{N}}_{[2],[1]}^{c=2}: \quad$\begin{tabular}{|c|cccccc|}
\hline$k \backslash n=$ & -1 & 1 & 3 & 5 & 7 & 9 \\
\hline 0 & 2 & -15 & 30 & -20 & 0 & 3 \\
1 & 1 & -11 & 31 & -22 & 0 & 1 \\
2 & 0 & -2 & 10 & -8 & 0 & 0 \\
3 & 0 & 0 & 1 & -1 & 0 & 0 \\
\hline
\end{tabular}

\begin{tabular}{c|c|ccccc|}
$\hat{\mathbf{N}}_{[1,1],[1]}^{c=0}$ & $k \backslash n=$ & -1 & 1 & 3 & 5 & 7 \\
\cline { 2 - 6 } & 0 & -12 & 40 & -48 & 24 & -4 \\
1 & -6 & 32 & -40 & 16 & -2 \\
2 & 0 & 6 & -8 & 2 & 0 \\
\hline
\end{tabular}

$\hat{\mathbf{N}}_{[1,1],[1]}^{c=1}: \quad$\begin{tabular}{|c|cccc|}
\hline$k \backslash n=$ & 0 & 2 & 4 & 6 \\
\hline 0 & 0 & 0 & 0 & 0 \\
1 & 21 & -15 & -1 & 3 \\
2 & 25 & -20 & 0 & 1 \\
3 & 9 & -8 & 0 & 0 \\
4 & 1 & -1 & 0 & 0 \\
\hline
\end{tabular}

$\hat{\mathbf{N}}_{[1,1],[1]}^{c=2}:$\begin{tabular}{|c|cccccc|}
\hline$k \backslash n=$ & -1 & 1 & 3 & 5 & 7 & 9 \\
\hline 0 & 6 & -21 & 26 & -12 & 0 & 1 \\
1 & 5 & -22 & 27 & -10 & 0 & 0 \\
2 & 1 & -8 & 9 & -2 & 0 & 0 \\
3 & 0 & -1 & 1 & 0 & 0 & 0 \\
\hline
\end{tabular}




\subsection{Racah matrices for links}

In this subsection, we write down the inclusive Racah matrices that are necessary in order to perform calculations for checking the integrality conjectures in the case of 2-component links in the previous subsection. Non-trivial (new) Racah matrices are required in the following cases:

HOMFLY polynomials with one component in the adjoint representation and the other one in the fundamental one. In this case, one studies the product of $\operatorname{SU}(N)$ representations

$$
[1] \otimes[1] \otimes([1],[1])=([3],[1])+2([2,1],[1])+2[2]+2[1,1]+([1,1,1],[1])
$$

and one can use the eigenvalue conjecture for links, [80] in order to construct the inclusive $2 \times 2$ matrices. Note that, since we are dealing here with links, there are two different matrices for each representation [80]. The eigenvalues (diagonalized $\mathcal{R}$-matrices, [73-76]) are

$$
\begin{array}{rlr}
\mathcal{R}_{([3],[1]) ; x x}=\left(A q^{-1}\right), & \mathcal{R}_{([1,1,1],[1]) ; x x}=(-A q), & \mathcal{R}_{([3],[1]) ; x y}=\mathcal{R}_{([1,1,1],[1]) ; x y}=(q) \\
\mathcal{R}_{([2,1],[1]) ; x x}=\left(\begin{array}{cc}
A q^{-1} & \\
& -A q
\end{array}\right), & \mathcal{R}_{([2,1],[1]) ; x y}=\left(\begin{array}{cc}
q \\
-q^{-1}
\end{array}\right), \\
\mathcal{R}_{[2] ; x x}=\left(\begin{array}{cc}
A q^{-1} & \\
-A q
\end{array}\right), & \mathcal{R}_{[2] ; x y}=\left(\begin{array}{c}
A \\
-q^{-1}
\end{array}\right), \\
\mathcal{R}_{[1,1] ; x x}=\left(\begin{array}{cc}
A q^{-1} & \\
& -A q
\end{array}\right), & \mathcal{R}_{[1,1] ; x y}=\left(\begin{array}{c}
A \\
q
\end{array}\right)
\end{array}
$$

and the mixing (inclusive Racah) matrices are

$$
\begin{array}{rlrl}
U_{([3],[1]) ; x x y} & =U_{([1,1,1],[1]) ; x x y}=U_{([3],[1]) ; x y x}=U_{([1,1,1],[1]) ; x y x}=(1) \\
U_{([2,1],[1]) ; x x y} & =\left(\begin{array}{cc}
-\frac{1}{[2]} & \frac{\sqrt{[3]}}{[2]} \\
\frac{\sqrt{[3]}}{[2]} & \frac{1}{[2]}
\end{array}\right), & U_{([2,1],[1]) ; x y x}=\left(\begin{array}{cc}
-\frac{1}{[2]} & -\frac{\sqrt{[3]}}{[2]} \\
-\frac{\sqrt{[3]}}{[2]} & \frac{1}{[2]}
\end{array}\right), \\
U_{[2] ; x x y} & =\left(\begin{array}{cc}
-\sqrt{\frac{D_{0}}{D_{1}[2]}} & \sqrt{\frac{D_{2}}{D_{1}[2]}} \\
\sqrt{\frac{D_{2}}{D_{1}[2]}} & \sqrt{\frac{D_{0}}{D_{1}[2]}}
\end{array}\right), & U_{[2] ; x y x}=\left(\begin{array}{cc}
-\frac{1}{D_{1}} & \frac{\sqrt{D_{2} D_{0}}}{D_{1}} \\
\frac{\sqrt{D_{2} D_{0}}}{D_{1}} & \frac{1}{D_{1}}
\end{array}\right), \\
U_{[1,1] ; x x y}=\left(\begin{array}{ccc}
-\sqrt{\frac{D_{-2}}{D_{-1}[2]}} & \sqrt{\frac{D_{0}}{D_{-1}[2]}} \\
\sqrt{\frac{D_{0}}{D_{-1}[2]}} & \sqrt{\frac{D_{-2}}{D_{-1}[2]}}
\end{array}\right), & U_{[1,1] ; x y x}=\left(\begin{array}{cc}
-\frac{1}{D_{-1}} & \frac{\sqrt{D_{-2} D_{0}}}{D_{-1}} \\
\frac{\sqrt{D_{-2} D_{0}}}{D_{-1}} & \frac{1}{D_{-1}}
\end{array}\right)
\end{array}
$$

where $D_{i}=\left(A q^{i}-A^{-1} q^{-i}\right) /\left(q-q^{-1}\right)$.

Kauffman polynomials in the fundamental representation. In this case (when the answers can be also found in [131]), one studies the product of $\mathrm{SO}(N)$ representations

$$
[1] \otimes[1] \otimes[1]=[3]+2[2,1]+[1,1,1]+3[1]
$$


and both the $2 \times 2$ and $3 \times 3$ inclusive Racah matrices (since all three representations are the same, there is only one matrix for each representation) can be obtained from the eigenvalue conjecture for knots, [78]. The eigenvalues are

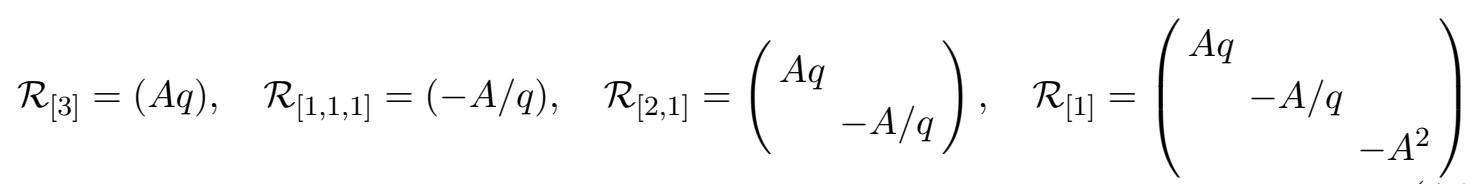

and, hence, the Racah matrices are

$$
\begin{aligned}
U_{[3]} & =U_{[1,1,1]}=(1) \\
U_{[2,1]} & =\frac{1}{[2]_{q}}\left(\begin{array}{cc}
1 & \sqrt{[3]_{q}} \\
\sqrt{[3]_{q}} & -1
\end{array}\right) \\
U_{[1]} & =\frac{1}{[2]_{q}}\left(\begin{array}{ccc}
\frac{A q+1}{A+q} & -\frac{1}{q} \sqrt{\frac{\left(A q^{3}-1\right)\left(A+q^{3}\right)}{(A q-1)(A+q)}} & \sqrt{[2]_{q} \frac{\left(A^{2}-1\right)\left(A+q^{3}\right)}{(A q-1)(A+q)}} \\
-\frac{1}{q} \sqrt{\frac{\left(A q^{3}-1\right)\left(A+q^{3}\right)}{(A q-1)(A+q)}} & \frac{A-q}{A q-1} & \sqrt{[2]_{q} \frac{\left(A^{2}-1\right)\left(A q^{3}-1\right)}{(A q-1)(A+q)}} \\
\sqrt{[2]_{q} \frac{\left(A^{2}-1\right)\left(A+q^{3}\right)}{(A q-1)(A+q)}} & \sqrt{[2]_{q} \frac{\left(A^{2}-1\right)\left(A q^{3}-1\right)}{(A q-1)(A+q)}} & {[2]_{q} A \frac{q^{2}-1}{(A q-1)(A+q)}}
\end{array}\right)
\end{aligned}
$$

where $[n]_{q}=\left(q^{n}-q^{-n}\right) /\left(q-q^{-1}\right)$ denotes the usual quantum number.

Kauffman polynomials with one component in the (anti)symmetric representation and the other one in the fundamental one. In this case, one studies the product of $\mathrm{SO}(N)$ representations

$$
[1] \otimes[1] \otimes[2]=[4]+2[3,1]+[2,2]+[2,1,1]+3[2]+2[1,1]+1 \text {. }
$$

The $2 \times 2$ inclusive Racah matrices can be again read off the eigenvalue conjecture, the eigenvalues being

$$
\begin{aligned}
& \mathcal{R}_{[4] ; x x}=\mathcal{R}_{[2,2] ; x x}=\mathcal{R}_{[\emptyset] ; x x}=\left(A q^{-1}\right), \quad \mathcal{R}_{[2,1,1] ; x x}=(-A q), \\
& \mathcal{R}_{[4] ; x y}=\left(-q^{-2}\right), \quad \mathcal{R}_{[2,2] ; x y}=\mathcal{R}_{[2,1,1] ; x y}=(q), \mathcal{R}_{[\emptyset] ; x y}=(-A q) \\
& \mathcal{R}_{[3,1] ; x x}=\left(\begin{array}{cc}
A q^{-1} & \\
& -A q
\end{array}\right), \quad \quad \mathcal{R}_{[3,1] ; x y}=\left(\begin{array}{ll}
-q^{-2} & \\
& -q
\end{array}\right), \\
& \mathcal{R}_{[1,1] ; x x}=\left(\begin{array}{cc}
A q^{-1} & \\
& -A q
\end{array}\right), \quad \quad \mathcal{R}_{[1,1] ; x y}=\left(\begin{array}{ll}
q & \\
& -A q
\end{array}\right) \\
& \mathcal{R}_{([2]) ; x x}=\left(\begin{array}{ccc}
A q^{-1} & & \\
& -A q & \\
& & A^{2}
\end{array}\right), \quad \quad \mathcal{R}_{([2]) ; x y}=\left(\begin{array}{ccc}
q^{-2} & & \\
& -q & \\
& & A q
\end{array}\right),
\end{aligned}
$$


and the mixing matrices being

$$
\begin{array}{ll}
U_{[3,1] ; x x y}=\left(\begin{array}{cc}
-\frac{1}{\sqrt{[3]}} & \sqrt{\frac{[4]}{[2][3]}} \\
\sqrt{\frac{[4]}{[2][3]}} & \frac{1}{\sqrt{[3]}}
\end{array}\right), \quad U_{[3,1] ; x y x}=\left(\begin{array}{cc}
-\frac{1}{\sqrt{[3]}} & -\frac{\sqrt{[2][4]}}{[3]} \\
-\frac{\sqrt{[2][4]}}{3]} & \frac{1}{\sqrt{[3]}}
\end{array}\right), \\
U_{[1,1] ; x x y}=\left(\begin{array}{cc}
-\sqrt{\frac{D_{-1}}{D_{0}[2]}} & \sqrt{\frac{D_{1}}{D_{0}[2]}} \\
\sqrt{\frac{D_{1}}{D_{0}[2]}} & \sqrt{\frac{D_{-1}}{D_{0}[2]}}
\end{array}\right), & U_{[1,1] ; x y x}=\left(\begin{array}{cc}
-\frac{1}{D_{0}} & -\frac{\sqrt{D_{1} D_{-1}}}{D_{0}} \\
-\frac{\sqrt{D_{1} D_{-1}}}{D_{0}} & \frac{1}{D_{0}}
\end{array}\right)
\end{array}
$$

while the $3 \times 3$ matrices ${ }^{4}$ are of the form

$$
\begin{aligned}
& U_{[2] ; x x y}=\left(\begin{array}{ccc}
\sqrt{\frac{(A-q) q}{\left(A q^{3}-1\right)[3]}} & \sqrt{\left(A+q^{3}\right)\left(A q^{5}-1\right)} A q^{4}\left(A-A^{-1}\right)[2][3] & \sqrt{\frac{\left(A q^{5}-1\right)(A q+1)(A-q)}{A q^{2}\left(A q^{3}-1\right)\left(A-A^{-1}\right)[2]}} \\
\sqrt{\frac{\left(A q^{5}-1\right)\left(A+q^{3}\right)}{q^{2}\left(A q^{3}-1\right)(A+q)[3]}} & \sqrt{\frac{\left(A q^{3}+1\right)^{2}(A-q)}{q^{3}(A+q)\left(A^{2}-1\right)[2][3]}} & -\sqrt{\frac{\left(A^{2} q^{2}-1\right)(A q-1)\left(A+q^{3}\right)}{q\left(A q^{3}-1\right)\left(A^{2}-1\right)(A+q)[2]}} \\
\sqrt{\frac{\left(A q^{5}-1\right)(A q+1)}{q\left(A q^{3}-1\right)(A+q)[3]}} & -\sqrt{\frac{(A q+1)\left(A+q^{3}\right)(A-q)[2]}{q^{2}\left(A^{2}-1\right)(A+q)[3]}} & \sqrt{\frac{A^{2}[2]\left(q^{2}-1\right)^{2}}{\left(A q^{3}-1\right)(A+q)\left(A^{2}-1\right)}}
\end{array}\right) \\
& U_{[2] ; x y x}=\left(\begin{array}{ccc}
\frac{q(A-q)}{\left(A q^{3}-1\right)[3]} & \sqrt{\frac{\left(A+q^{3}\right)(A-q)\left(A q^{5}-1\right)[2]}{q^{3}\left(A q^{3}-1\right)\left(A^{2}-1\right)[3]^{2}}} & \sqrt{\frac{(A q-1)\left(A q^{5}-1\right)\left(A^{2} q^{2}-1\right)[2]}{q\left(A q^{3}-1\right)^{2}\left(A^{2}-1\right)[3]}} \\
\sqrt{\frac{\left(A+q^{3}\right)(A-q)\left(A q^{5}-1\right)[2]}{q^{3}\left(A q^{3}-1\right)\left(A^{2}-1\right)[3]^{2}}} & \frac{A^{2} q^{4}+A^{2} q^{2}-A q^{3}+A q-q^{2}-1}{q^{2}\left(A^{2}-1\right)[3]} & -\sqrt{\frac{(A-q)(A q-1)\left(A^{2} q^{2}-1\right)\left(A+q^{3}\right)}{q^{2}\left(A q^{3}-1\right)\left(A^{2}-1\right)^{2}[3]}} \\
\sqrt{\frac{(A q-1)\left(A q^{5}-1\right)\left(A^{2} q^{2}-1\right)[2]}{q\left(A q^{3}-1\right)^{2}\left(A^{2}-1\right)[3]}} & -\sqrt{\frac{(A-q)(A q-1)\left(A^{2} q^{2}-1\right)\left(A+q^{3}\right)}{q^{2}\left(A q^{3}-1\right)\left(A^{2}-1\right)^{2}[3]}} & \frac{A(A-q)\left(q^{2}-1\right)}{\left(A q^{3}-1\right)\left(A^{2}-1\right)}
\end{array}\right) .
\end{aligned}
$$

\section{Gaussian distributions of the LMOV numbers $N$}

In this section, we make clear a specific behaviour of the LMOV numbers that can be understood from analyses of results of sections 4 and 4.2. Namely, we plot the numbers for the knot $8_{20}$ from our tables in sections 4 and 4.2 against $k$. From these pictures, one can easily see that these numbers have Gaussian distributions(!). One can also see that the difference with the Gaussian distribution decreases with the size of the representation (since in the $\mathrm{SO} / \mathrm{Sp}$ case only the first symmetric representation is available, the accuracy is lower in this case). This is a simplest corollary of the results obtained in this paper, its theoretical meaning and implications of this spectacular fact as well as other applications of the results obtained in this paper will be discussed elsewhere.

Thus, it turns out that with a very good accuracy for any given knot $\mathcal{K}$, representation $Q$ and parameter $n$, the LMOV numbers as a function of remaining variable $k$ are described by the formula

$$
N_{Q, n, k}^{\mathcal{K}} \approx G_{Q, n}^{\mathcal{K}}(k) \equiv \frac{I_{Q, n}^{\mathcal{K}}}{\sqrt{2 \pi} \sigma_{Q, n}^{\mathcal{K}}} \exp \left(-\frac{\left(k-\mu_{Q, n}^{\mathcal{K}}\right)^{2}}{2 \sigma_{Q, n}^{2}}\right)
$$

\footnotetext{
${ }^{4}$ Due to the relations between $\mathrm{SO}(N)$ and $\mathrm{SU}(N)$ theories particular cases of these matrices can be used for calculations of HOMFLY polynomials. Namely, due to similarity between $\mathrm{SU}(2)$ and $\mathrm{SO}(3)$ groups by substituting $A=q^{4}$ and $q=q^{2}$ one gets matrix $3 \times 3$ for representation [6,2] from the tensor product $[2] \otimes[2] \otimes[4]$. And due to similarity between $\mathrm{SO}(6)$ and $\mathrm{SU}(4)$ by substituting $A=q^{5}$ one gets matrix $3 \times 3$ for representation $[3,3,1,1]$ from the tensor product $[1,1] \otimes[1,1] \otimes[2,2]$.
} 


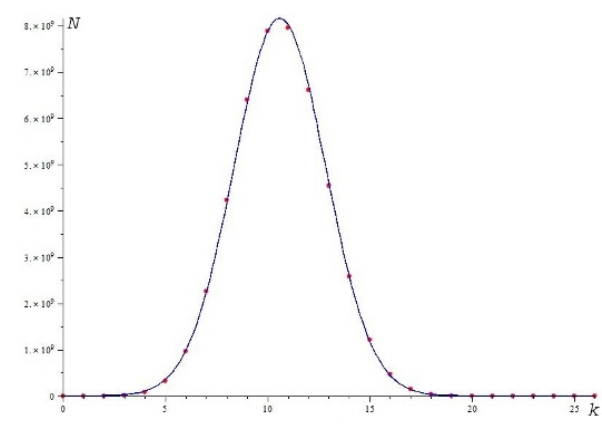

Figure 1. Red dots are $N_{[4],-20, k}$ numbers, blue curve is a Gaussian distribution with parameters $\mu=10.6, \sigma=2.24, I=4.56 \cdot 10^{10}$.

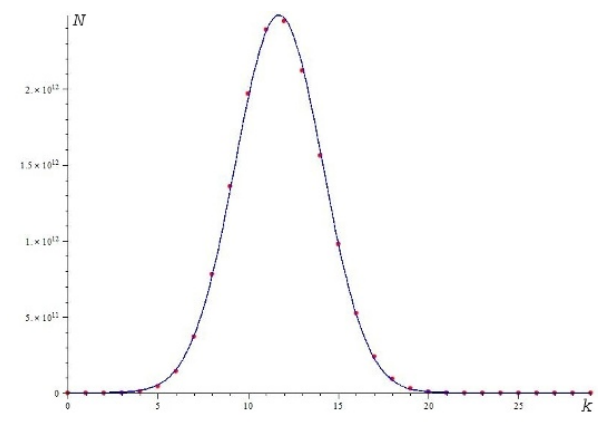

Figure 2. Red dots are $N_{[3,1],-16, k}$ numbers, blue curve is a Gaussian distribution with parameters $\mu=11.7, \sigma=2.42, I=1.51 \cdot 10^{13}$.

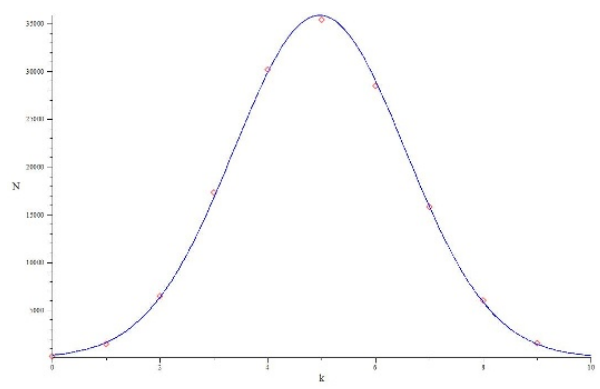

Figure 3. Red dots are $\hat{N}_{[2],-11, k}^{c=1}$ numbers, blue curve is a Gaussian distribution with parameters $\mu=4.96 \sigma=1.59 I=1.43 \cdot 10^{5}$.

with only three parameters $\mu, \sigma$ and $I$ which can depend on $\mathcal{K}, Q$ and $n$, but not on $k$. Accuracy of this formula is illustrated in figures 1 and 2 for the LMOV invariants in the $\mathrm{SU}(N)$ case and 3, 4 for the LMOV invariants in the SO/Sp case:

\section{Conclusion}

In this paper, we reported a positive result of new tests of the LMOV integrality conjectures, made possible by a recent progress in evaluation of the colored knot polynomials and first 


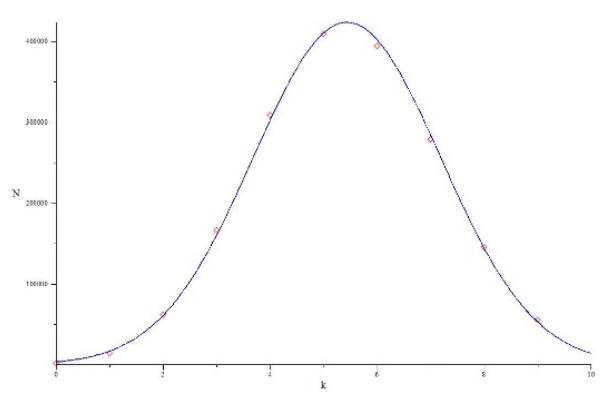

Figure 4. Red dots are $\hat{N}_{[1,1],-7, k}^{c=1}$ numbers, blue curve is a Gaussian distribution with parameters $\mu=5.44 \sigma=1.75 I=1.8510^{6}$.

corollary of the performed calculations: the Gaussian distribution of the LMOV invariants. The progress is a cumulative effect of merging of the different research directions:

i) reformulation of the RT formalism in the spaces of intertwining operators [73-76][80] with developments of the highest weight technique [31, 33] and the eigenvalue conjecture $[1,78]$ to evaluate the inclusive and exclusive Racah matrices [33]-[35],

ii) representing the knot polynomials for all arborescent knots through the exclusive Racah matrices $S$ and $\bar{S}$ [29],

iii) developing the family technique $[30,32,144,145]$ in order to adequately classify knots, at least, for calculational purposes,

iv) applying Vogel's universality [154] to handle the adjoint representations and their descendants; important here is that deviations from the universality at the group theory level are not seen in knot polynomial calculus [1, 141].

The work in all these directions is hard, but interesting and important (see also a new development in another direction of an integrality conjecture for superpolynomials, [155]). The integrality tests are a non-trivial application of its results, and provide an additional stimulus for new advances.

\section{Acknowledgments}

Our work is partly supported by RFBR grants 16-01-00291 (A.Mir.), 16-02-01021 (A.Mor.), mol-a-dk 16-32-60047 (An.Mor), mol-a-dk 16-31-60082 and MK-8769.2016.1 (A.S.) and by joint grants 17-51-50051-YaF, 15-51-52031-NSC-a, 16-51-45029-Ind-a and 16-51-53034GFEN.

Open Access. This article is distributed under the terms of the Creative Commons Attribution License (CC-BY 4.0), which permits any use, distribution and reproduction in any medium, provided the original author(s) and source are credited. 


\section{References}

[1] A. Mironov and A. Morozov, Universal Racah matrices and adjoint knot polynomials: Arborescent knots, Phys. Lett. B 755 (2016) 47 [arXiv:1511.09077] [INSPIRE].

[2] R. Gopakumar and C. Vafa, On the gauge theory/geometry correspondence, Adv. Theor. Math. Phys. 3 (1999) 1415 [hep-th/9811131] [INSPIRE].

[3] R. Gopakumar and C. Vafa, M theory and topological strings - II, hep-th/9812127 [INSPIRE].

[4] H. Ooguri and C. Vafa, Knot invariants and topological strings, Nucl. Phys. B 577 (2000) 419 [hep-th/9912123] [INSPIRE].

[5] S.-S. Chern and J. Simons, Characteristic forms and geometric invariants, Annals Math. 99 (1974) 48 [INSPIRE].

[6] E. Witten, Quantum Field Theory and the Jones Polynomial, Commun. Math. Phys. 121 (1989) 351 [INSPIRE].

[7] J.W. Alexander, Topological invariants of knots and links, Trans. Am. Math. Soc. 30 (1928) 275.

[8] V.F.R. Jones, Index for subfactors, Invent. Math. 72 (1983) 1 [INSPIRE].

[9] V.F.R. Jones, A polynomial invariant for knots via von Neumann algebras, Bull. Am. Math. Soc. 12 (1985) 103 [INSPIRE].

[10] V.F.R. Jones, Hecke algebra representations of braid groups and link polynomials, Annals Math. 126 (1987) 335 [INSPIRE].

[11] L. Kauffman, State models and the Jones polynomial, Topology 26 (1987) 395.

[12] P. Fréyd, D. Yetter, J. Hoste, W.B.R. Lickorish, K. Millett and A. Ocneanu, A new polynomial invariant of knots and links, Bull. Am. Math. Soc. 12 (1985) 239 [INSPIRE].

[13] J.H. Przytycki and K.P. Traczyk, Invariants of Conway type, Kobe J. Math. 4 (1987) 115

[14] J.H. Conway, An Enumeration of Knots and Links, and Some of Their Algebraic Properties, in Computational Problems in Abstract Algebra, J. Leech ed., Pergamon Press Ltd., Oxford U.K. (1970), pp. 329-358 [https://doi.org/10.1016/B978-0-08-012975-4.50034-5].

[15] J.M.F. Labastida and M. Mariño, Polynomial invariants for torus knots and topological strings, Commun. Math. Phys. 217 (2001) 423 [hep-th/0004196] [INSPIRE].

[16] J.M.F. Labastida, M. Mariño and C. Vafa, Knots, links and branes at large-N, JHEP 11 (2000) 007 [hep-th/0010102] [INSPIRE].

[17] J.M.F. Labastida and M. Mariño, A New point of view in the theory of knot and link invariants, math/0104180 [INSPIRE].

[18] M. Mariño and C. Vafa, Framed knots at large-N, Contemp. Math. 310 (2002) 185 [hep-th/0108064] [INSPIRE].

[19] S. Garoufalidis, P. Kucharski and P. Sułkowski, Knots, BPS states and algebraic curves, Commun. Math. Phys. 346 (2016) 75 [arXiv:1504.06327] [INSPIRE].

[20] P. Kucharski and P. Sułkowski, BPS counting for knots and combinatorics on words, JHEP 11 (2016) 120 [arXiv:1608.06600] [INSPIRE]. 
[21] W. Luo and S. Zhu, Integrality structures in topological strings I: framed unknot, arXiv:1611.06506 [INSPIRE].

[22] P. Ramadevi, T.R. Govindarajan and R.K. Kaul, Chirality of knots $9_{42}$ and $10_{71}$ and Chern-Simons theory, Mod. Phys. Lett. A 9 (1994) 3205 [hep-th/9401095] [INSPIRE].

[23] S. Nawata, P. Ramadevi and Zodinmawia, Colored HOMFLY polynomials from Chern-Simons theory, J. Knot Theory Ramifications 22 (2013) 1350078 [arXiv:1302.5144] [INSPIRE].

[24] Zodinmawia, Knot polynomials from $\mathrm{SU}(N)$ Chern-Simons theory, superpolynomials and super-A-polynomials, Ph.D. Thesis, IIT, Mumbai India (2014).

[25] D. Galakhov, D. Melnikov, A. Mironov, A. Morozov and A. Sleptsov, Colored knot polynomials for arbitrary pretzel knots and links, Phys. Lett. B 743 (2015) 71 [arXiv:1412.2616] [INSPIRE].

[26] A. Mironov, A. Morozov and A. Sleptsov, Colored HOMFLY polynomials for the pretzel knots and links, JHEP 07 (2015) 069 [arXiv:1412.8432] [INSPIRE].

[27] D. Galakhov, D. Melnikov, A. Mironov and A. Morozov, Knot invariants from Virasoro related representation and pretzel knots, Nucl. Phys. B 899 (2015) 194 [arXiv:1502.02621] [INSPIRE].

[28] S. Nawata, P. Ramadevi and V.K. Singh, Colored HOMFLY polynomials that distinguish mutant knots, arXiv:1504.00364 [INSPIRE].

[29] A. Mironov, A. Morozov, A. Morozov, P. Ramadevi and V.K. Singh, Colored HOMFLY polynomials of knots presented as double fat diagrams, JHEP 07 (2015) 109 [arXiv: 1504.00371] [INSPIRE].

[30] A. Mironov and A. Morozov, Towards effective topological field theory for knots, Nucl. Phys. B 899 (2015) 395 [arXiv: 1506.00339] [INSPIRE].

[31] A. Mironov, A. Morozov, A. Morozov and A. Sleptsov, Colored knot polynomials: HOMFLY in representation [2,1], Int. J. Mod. Phys. A 30 (2015) 1550169 [arXiv: 1508.02870] [INSPIRE].

[32] A. Mironov, A. Morozov, A. Morozov, P. Ramadevi, V.K. Singh and A. Sleptsov, Tabulating knot polynomials for arborescent knots, J. Phys. A 50 (2017) 085201 [arXiv: 1601.04199] [INSPIRE].

[33] A. Mironov, A. Morozov, A. Morozov and A. Sleptsov, HOMFLY polynomials in representation [3,1] for 3-strand braids, JHEP 09 (2016) 134 [arXiv: 1605.02313] [INSPIRE].

[34] A. Mironov, A. Morozov, A. Morozov and A. Sleptsov, Quantum Racah matrices and 3-strand braids in irreps $R$ with $|R|=4$, JETP Lett. 104 (2016) 56 [Pisma Zh. Eksp. Teor. Fiz. 104 (2016) 52] [arXiv: 1605.03098] [INSPIRE].

[35] A. Mironov, A. Morozov, A. Morozov and A. Sleptsov, Racah matrices and hidden integrability in evolution of knots, Phys. Lett. B 760 (2016) 45 [arXiv:1605.04881] [INSPIRE].

[36] http://knotebook.org.

[37] M. Khovanov, A categorification of the Jones polynomial, Duke Math. J. 101 (2000) 359 [math/9908171] [INSPIRE]. 
[38] M. Khovanov, Patterns in knot cohomology I, Exp. Math. 12 (2003) 365 [math/0201306].

[39] M. Khovanov, Categorifications of the colored Jones polynomial, J. Knot Theory Ramifications 14 (2005) 111 [math/0302060].

[40] M. Khovanov, sl(3) link homology, Algebr. Geom. Topol. 4 (2004) 1045 [math/0304375].

[41] M. Khovanov, Triply-graded link homology and Hochschild homology of Soergel bimodules, Int. J. Math. 18 (2007) 869 [math/0510265].

[42] M. Khovanov, Link homology and categorification, math/0605339.

[43] M. Khovanov, Categorifications from planar diagrammatics, arXiv:1008.5084.

[44] D. Bar-Natan, On Khovanov's categorification of the Jones polynomial, Algebr. Geom. Topol. 2 (2002) 337 [math/0201043].

[45] D. Bar-Natan, Khovanov's homology for tangles and cobordisms, Geom. Topol. 9 (2005) 1443 [math/0410495].

[46] D. Bar-Natan, Fast Khovanov Homology Computations, J. Knot Theory Ramifications 16 (2007) 243 [math/0606318].

[47] M. Khovanov and L. Rozansky, Matrix factorizations and link homology, Fund. Math. 199 (2008) 191 [math/0401268].

[48] M. Khovanov and L. Rozansky, Matrix factorizations and link homology II, Geom. Topol. 12 (2008) 1387 [math/0505056].

[49] M. Khovanov and L. Rozansky, Virtual crossings, convolutions and a categorification of the $\mathrm{SO}(2 N)$ Kauffman polynomial, math/0701333.

[50] N. Carqueville and D. Murfet, Computing Khovanov-Rozansky homology and defect fusion, Algebr. Geom. Topol. 14 (2014) 489 [arXiv:1108.1081] [INSPIRE].

[51] V. Dolotin and A. Morozov, Introduction to Khovanov Homologies. I. Unreduced Jones superpolynomial, JHEP 01 (2013) 065 [arXiv: 1208.4994] [INSPIRE].

[52] V. Dolotin and A. Morozov, Introduction to Khovanov Homologies. II. Reduced Jones superpolynomials, J. Phys. Conf. Ser. 411 (2013) 012013 [arXiv:1209.5109] [INSPIRE].

[53] V. Dolotin and A. Morozov, Introduction to Khovanov Homologies. III. A new and simple tensor-algebra construction of Khovanov-Rozansky invariants, Nucl. Phys. B 878 (2014) 12 [arXiv:1308.5759] [INSPIRE].

[54] A. Anokhina and A. Morozov, Towards R-matrix construction of Khovanov-Rozansky polynomials. I. Primary T-deformation of HOMFLY, JHEP 07 (2014) 063 [arXiv: 1403.8087] [INSPIRE].

[55] S. Nawata and A. Oblomkov, Lectures on knot homology, Contemp. Math. 680 (2016) 137 [arXiv: 1510.01795] [InSPIRE].

[56] D. Galakhov and G.W. Moore, Comments On The Two-Dimensional Landau-Ginzburg Approach To Link Homology, arXiv: 1607.04222 [INSPIRE].

[57] S. Gukov, D. Pei, P. Putrov and C. Vafa, BPS spectra and 3-manifold invariants, arXiv: 1701.06567 [INSPIRE].

[58] N.Y. Reshetikhin and V.G. Turaev, Ribbon graphs and their invariants derived from quantum groups, Commun. Math. Phys. 127 (1990) 1 [INSPIRE]. 
[59] E. Guadagnini, M. Martellini and M. Mintchev, Chern-Simons field theory and quantum groups, in proceedings of Quantum Groups. 8th International Workshop on Mathematical Physics, Clausthal, Germany, 19-26 July 1989, pp. 307-317

[https://doi.org/10.1007/3-540-53503-9_51].

[60] E. Guadagnini, M. Martellini and M. Mintchev, Chern-Simons Holonomies and the Appearance of Quantum Groups, Phys. Lett. B 235 (1990) 275 [InSPIRE].

[61] V.G. Turaev and O.Y. Viro, State sum invariants of 3-manifolds and quantum 6j-symbols, Topology 31 (1992) 865 [INSPIRE].

[62] A. Morozov and A. Smirnov, Chern-Simons Theory in the Temporal Gauge and Knot Invariants through the Universal Quantum R-Matrix, Nucl. Phys. B 835 (2010) 284 [arXiv: 1001.2003] [INSPIRE].

[63] A. Smirnov, Notes on Chern-Simons Theory in the Temporal Gauge, in proceedings of the 47 th International School of Subnuclear Physics: The most unexepted at LHC and the status of high energy frontier (ISSP 2009), Erice, Sicily, Italy, 29 August-7 September 2009 [Subnucl. Ser. 47 (2011) 489] [arXiv:0910.5011] [INSPIRE].

[64] R.K. Kaul and T.R. Govindarajan, Three-dimensional Chern-Simons theory as a theory of knots and links, Nucl. Phys. B 380 (1992) 293 [hep-th/9111063] [INSPIRE].

[65] R.K. Kaul and T.R. Govindarajan, Three-dimensional Chern-Simons theory as a theory of knots and links. 2. Multicolored links, Nucl. Phys. B 393 (1993) 392 [INSPIRE].

[66] P. Ramadevi, T.R. Govindarajan and R.K. Kaul, Three-dimensional Chern-Simons theory as a theory of knots and links. 3. Compact semisimple group, Nucl. Phys. B 402 (1993) 548 [hep-th/9212110] [INSPIRE].

[67] P. Ramadevi, T.R. Govindarajan and R.K. Kaul, Knot invariants from rational conformal field theories, Nucl. Phys. B 422 (1994) 291 [hep-th/9312215] [InSPIRE].

[68] P. Ramadevi, T.R. Govindarajan and R.K. Kaul, Representations of composite braids and invariants for mutant knots and links in Chern-Simons field theories, Mod. Phys. Lett. A 10 (1995) 1635 [hep-th/9412084] [INSPIRE].

[69] P. Ramadevi and T. Sarkar, On link invariants and topological string amplitudes, Nucl. Phys. B 600 (2001) 487 [hep-th/0009188] [INSPIRE].

[70] P. Borhade, P. Ramadevi and T. Sarkar, U(N) framed links, three manifold invariants and topological strings, Nucl. Phys. B 678 (2004) 656 [hep-th/0306283] [INSPIRE].

[71] P. Ramadevi and Zodinmawia, $\mathrm{SU}(N)$ quantum Racah coefficients and non-torus links, Nucl. Phys. B 870 (2013) 205 [arXiv:1107.3918] [inSPIRE].

[72] P. Ramadevi and Zodinmawia, Reformulated invariants for non-torus knots and links, arXiv:1209.1346 [INSPIRE].

[73] A. Mironov, A. Morozov and A. Morozov, Character expansion for HOMFLY polynomials. I. Integrability and difference equations, in Strings, Gauge Fields, and the Geometry Behind: The Legacy of Maximilian Kreuzer, A. Rebhan, L. Katzarkov, J. Knapp, R. Rashkov and E. Scheidegger eds., World Scietific Publishins Co. Pte. Ltd. (2013), pp. 101-118 [arXiv:1112.5754] [INSPIRE].

[74] A. Mironov, A. Morozov and A. Morozov, Character expansion for HOMFLY polynomials. II. Fundamental representation. Up to five strands in braid, JHEP 03 (2012) 034 [arXiv:1112.2654] [INSPIRE]. 
[75] A. Anokhina, A. Mironov, A. Morozov and A. Morozov, Racah coefficients and extended HOMFLY polynomials for all 5-, 6- and 7-strand braids, Nucl. Phys. B 868 (2013) 271 [arXiv: 1207.0279] [INSPIRE].

[76] A. Anokhina, On R-matrix approaches to knot invariants, arXiv:1412.8444 [INSPIRE].

[77] H. Itoyama, A. Mironov, A. Morozov and A. Morozov, Character expansion for HOMFLY polynomials. III. All 3-Strand braids in the first symmetric representation, Int. J. Mod. Phys. A 27 (2012) 1250099 [arXiv:1204.4785] [INSPIRE].

[78] H. Itoyama, A. Mironov, A. Morozov and A. Morozov, Eigenvalue hypothesis for Racah matrices and HOMFLY polynomials for 3-strand knots in any symmetric and antisymmetric representations, Int. J. Mod. Phys. A 28 (2013) 1340009 [arXiv:1209.6304] [INSPIRE].

[79] A. Anokhina, A. Mironov, A. Morozov and A. Morozov, Colored HOMFLY polynomials as multiple sums over paths or standard Young tableaux, Adv. High Energy Phys. 2013 (2013) 931830 [arXiv: 1304.1486] [INSPIRE].

[80] A. Anokhina and A. Morozov, Cabling procedure for the colored HOMFLY polynomials, Theor. Math. Phys. 178 (2014) 1 [Teor. Mat. Fiz. 178 (2014) 3] [arXiv:1307.2216] [INSPIRE].

[81] E. Witten, Supersymmetry and Morse theory, J. Diff. Geom. 17 (1982) 661 [InSPIRE].

[82] A. Kapustin and E. Witten, Electric-Magnetic Duality And The Geometric Langlands Program, Commun. Num. Theor. Phys. 1 (2007) 1 [hep-th/0604151] [InSPIRE].

[83] E. Witten, Khovanov Homology And Gauge Theory, in Proceedings of the Freedman Fest, R. Kirby, V. Krushkal and Z. Wang eds., Mathematical Sciences Publishers (2012) [Geom. Topol. Monographs 18 (2012) 291] [arXiv:1108.3103] [INSPIRE].

[84] E. Witten, Two Lectures On The Jones Polynomial And Khovanov Homology, arXiv:1401.6996 [INSPIRE].

[85] E. Witten, Two Lectures on Gauge Theory and Khovanov Homology, arXiv: 1603.03854 [INSPIRE].

[86] D.E. Littlewood, The theory of group characters and matrix representations of groups, AMS Chelsea Publishing, AMS, Providence Rhode Island U.S.A. (1958).

[87] P. Dunin-Barkowski, A. Mironov, A. Morozov, A. Sleptsov and A. Smirnov, Superpolynomials for toric knots from evolution induced by cut-and-join operators, JHEP 03 (2013) 021 [arXiv: 1106.4305] [INSPIRE].

[88] A. Mironov, A. Morozov and S. Shakirov, Torus HOMFLY as the Hall-Littlewood Polynomials, J. Phys. A 45 (2012) 355202 [arXiv:1203.0667] [INSPIRE].

[89] J.J. Duistermaat and G. Heckman, On the variation in the cohomology of the symplectic form of the reduced phase space, Invent. Math. 69 (1982) 259 [Addendum ibid. 72 (1983) 153] [INSPIRE].

[90] M. Blau, E. Keski-Vakkuri and A.J. Niemi, Path integrals and geometry of trajectories, Phys. Lett. B 246 (1990) 92 [INSPIRE].

[91] A. Morozov, A.J. Niemi and K. Palo, Supersymmetry and loop space geometry, Phys. Lett. B 271 (1991) 365 [INSPIRE].

[92] A. Hietamaki, A. Morozov, A.J. Niemi and K. Palo, Geometry of $N=1 / 2$ supersymmetry and the Atiyah-Singer index theorem, Phys. Lett. B 263 (1991) 417 [InSPIRE]. 
[93] A.S. Schwarz and O. Zaboronsky, Supersymmetry and localization, Commun. Math. Phys. 183 (1997) 463 [hep-th/9511112] [INSPIRE].

[94] C. Beasley and E. Witten, Non-Abelian localization for Chern-Simons theory, J. Diff. Geom. 70 (2005) 183 [hep-th/0503126] [INSPIRE].

[95] J. Kallen, Cohomological localization of Chern-Simons theory, JHEP 08 (2011) 008 [arXiv: 1104.5353] [INSPIRE].

[96] V. Pestun, Localization of gauge theory on a four-sphere and supersymmetric Wilson loops, Commun. Math. Phys. 313 (2012) 71 [arXiv:0712.2824] [INSPIRE].

[97] A. Alexandrov, A. Mironov and A. Morozov, Instantons and merons in matrix models, Physica D 235 (2007) 126 [hep-th/0608228] [INSPIRE].

[98] A. Alexandrov, A. Mironov and A. Morozov, BGWM as Second Constituent of Complex Matrix Model, JHEP 12 (2009) 053 [arXiv:0906.3305] [INSPIRE].

[99] B. Eynard and N. Orantin, Invariants of algebraic curves and topological expansion, Commun. Num. Theor. Phys. 1 (2007) 347 [math-ph/0702045] [INSPIRE].

[100] N. Orantin, Symplectic invariants, Virasoro constraints and Givental decomposition, arXiv: 0808.0635 [INSPIRE].

[101] M. Tierz, Soft matrix models and Chern-Simons partition functions, Mod. Phys. Lett. A 19 (2004) 1365 [hep-th/0212128] [INSPIRE].

[102] A. Brini, B. Eynard and M. Mariño, Torus knots and mirror symmetry, Annales Henri Poincaré 13 (2012) 1873 [arXiv: 1105.2012] [INSPIRE].

[103] A. Aleksandrov, A. Mironov, A. Morozov and A. Morozov, Towards matrix model representation of HOMFLY polynomials, JETP Lett. 100 (2014) 271 [Pisma Zh. Eksp. Teor. Fiz. 100 (2014) 297] [arXiv:1407.3754] [INSPIRE].

[104] A. Alexandrov and D. Melnikov, Matrix integral expansion of colored Jones polynomials for figure-eight knot, JETP Lett. 101 (2015) 51 [Pisma Zh. Eksp. Teor. Fiz. 101 (2015) 54] [arXiv: 1411.5698] [INSPIRE].

[105] R. Dijkgraaf, H. Fuji and M. Manabe, The Volume Conjecture, Perturbative Knot Invariants and Recursion Relations for Topological Strings, Nucl. Phys. B 849 (2011) 166 [arXiv: 1010.4542$]$ [INSPIRE].

[106] A. Mironov, A. Morozov and A. Sleptsov, Genus expansion of HOMFLY polynomials, Theor. Math. Phys. 177 (2013) 1435 [Teor. Mat. Fiz. 177 (2013) 179] [arXiv:1303.1015] [INSPIRE].

[107] A. Mironov, A. Morozov and A. Sleptsov, On genus expansion of knot polynomials and hidden structure of Hurwitz tau-functions, Eur. Phys. J. C 73 (2013) 2492 [arXiv: 1304.7499] [INSPIRE].

[108] A. Mironov, A. Morozov, A. Sleptsov and A. Smirnov, On genus expansion of superpolynomials, Nucl. Phys. B 889 (2014) 757 [arXiv:1310.7622] [INSPIRE].

[109] A. Sleptsov, Hidden structures of knot invariants, Int. J. Mod. Phys. A 29 (2014) 1430063 [INSPIRE].

[110] A. Mironov, A. Morozov and S. Natanzon, Complete Set of Cut-and-Join Operators in Hurwitz-Kontsevich Theory, Theor. Math. Phys. 166 (2011) 1 [arXiv:0904.4227] [INSPIRE]. 
[111] A. Mironov, A. Morozov and S. Natanzon, Algebra of differential operators associated with Young diagrams, J. Geom. Phys. 62 (2012) 148 [arXiv:1012.0433] [INSPIRE].

[112] A. Alexandrov, A. Mironov, A. Morozov and S. Natanzon, Integrability of Hurwitz Partition Functions. I. Summary, J. Phys. A 45 (2012) 045209 [arXiv:1103.4100] [inSPIRE].

[113] A. Alexandrov, A. Mironov, A. Morozov and S. Natanzon, On KP-integrable Hurwitz functions, JHEP 11 (2014) 080 [arXiv:1405.1395] [INSPIRE].

[114] A. Mironov, A. Morozov and S. Natanzon, Integrability properties of Hurwitz partition functions. II. Multiplication of cut-and-join operators and WDVV equations, JHEP 11 (2011) 097 [arXiv: 1108. 0885] [INSPIRE].

[115] W.Fulton, Young tableaux: with applications to representation theory and geometry, London Mathematical Society (1997).

[116] R. Dijkgraaf, Mirror symmetry and elliptic curves, in The moduli spaces of curves, volume 129, Progress in Mathematics Series, Birkhäuser, Boston U.S.A. (1995), pp. 149-163.

[117] A. Okounkov, Toda equations for Hurwitz numbers, Math. Res. Lett. 7 (2000) 447 [math/0004128] [INSPIRE].

[118] A. Mironov, A. Morozov, S. Shakirov and A. Smirnov, Proving AGT conjecture as HS duality: extension to five dimensions, Nucl. Phys. B 855 (2012) 128 [arXiv:1105.0948] [INSPIRE].

[119] http://knotebook.org.s3-website-us-west-2.amazonaws.com/knotebook/HOMFLY/ colored.htm.

[120] http://knotebook.org.s3-website-us-west-2.amazonaws.com/knotebook/HOMFLY/ kauffman.htm.

[121] http://knotebook.org.s3-website-us-west-2.amazonaws.com/knotebook/HOMFLY/ universal.htm.

[122] K. Liu and P. Peng, Proof of the Labastida-Mariño-Ooguri-Vafa conjecture, J. Diff. Geom. 85 (2010) 479 [arXiv:0704.1526] [INSPIRE].

[123] K. Liu and P. Peng, On a proof of the Labastida-Mariño-Ooguri-Vafa conjecture, Math. Res. Lett. 17 (2010) 493 [arXiv: 1012.2635] [INSPIRE].

[124] M. Mariño, String theory and the Kauffman polynomial, Commun. Math. Phys. 298 (2010) 613 [arXiv:0904.1088] [INSPIRE].

[125] S. Stevan, Chern-Simons Invariants of Torus Links, Annales Henri Poincaré 11 (2010) 1201 [arXiv: 1003.2861] [INSPIRE].

[126] C. Paul, P. Borhade and P. Ramadevi, Composite Invariants and Unoriented Topological String Amplitudes, arXiv: 1003.5282 [INSPIRE].

[127] C. Paul, P. Borhade and P. Ramadevi, Composite Representation Invariants and Unoriented Topological String Amplitudes, Nucl. Phys. B 841 (2010) 448 [arXiv:1008.3453] [InSPIRE].

[128] S. Nawata, P. Ramadevi and Zodinmawia, Colored Kauffman Homology and Super-A-polynomials, JHEP 01 (2014) 126 [arXiv:1310.2240] [INSPIRE].

[129] A. Caudron, Classification des noeuds et des enlacements, volume 82-4, Publications mathématiques d'Orsay Series, Département de Mathématiques d'Orsay - Université Paris-Sud, Orsay France (1982). 
[130] F. Bonahon and L.C. Siebenmann, New geometric splittings of classical knots and the classification and symmetries of arborescent knots, (2016) http://www-bcf.usc.edu/ fbonahon/Research/Preprints/BonSieb.pdf.

[131] D. Bar-Natan and S. Morrison, Main Page. The Knot Atlas, (2015) http://katlas.org.

[132] M. Aganagic and C. Vafa, Mirror symmetry, D-branes and counting holomorphic discs, hep-th/0012041 [INSPIRE].

[133] S. Sinha and C. Vafa, SO and Sp Chern-Simons at large-N, hep-th/0012136 [INSPIRE].

[134] V. Bouchard, B. Florea and M. Mariño, Counting higher genus curves with crosscaps in Calabi-Yau orientifolds, JHEP 12 (2004) 035 [hep-th/0405083] [INSPIRE].

[135] V. Bouchard, B. Florea and M. Mariño, Topological open string amplitudes on orientifolds, JHEP 02 (2005) 002 [hep-th/0411227] [INSPIRE].

[136] P. Borhade and P. Ramadevi, $\mathrm{SO}(N)$ reformulated link invariants from topological strings, Nucl. Phys. B 727 (2005) 471 [hep-th/0505008] [INSPIRE].

[137] L. Rudolph, A congruence between link polynomials, Math. Proc. Cambridge Philos. Soc. 107 (1990) 319.

[138] H.R. Morton, Integrality of Homfly 1-tangle invariants, Algebr. Geom. Topol. 7 (2007) 327.

[139] H.R. Morton and N.D.A. Ryder, Relations between Kauffman and Homfly satellite invariants, Math. Proc. Cambridge Philos. Soc. 149 (2010) 105 [arXiv:0902.1339].

[140] K. Koike, On the decomposition of tensor products of the representations of the classical groups: by means of the universal characters, Adv. Math. 74 (1989) 57.

[141] A. Mironov, R. Mkrtchyan and A. Morozov, On universal knot polynomials, JHEP 02 (2016) 078 [arXiv:1510.05884] [INSPIRE].

[142] N.M. Dunfield, S. Gukov and J. Rasmussen, The Superpolynomial for knot homologies, Exp. Math. 15 (2006) 129 [math/0505662] [INSPIRE].

[143] H. Itoyama, A. Mironov, A. Morozov and A. Morozov, HOMFLY and superpolynomials for figure eight knot in all symmetric and antisymmetric representations, JHEP 07 (2012) 131 [arXiv:1203.5978] [INSPIRE].

[144] A. Mironov, A. Morozov and A. Morozov, Evolution method and "differential hierarchy" of colored knot polynomials, AIP Conf. Proc. 1562 (2013) 123 [arXiv:1306.3197] [InSPIRE].

[145] S. Arthamonov, A. Mironov, A. Morozov and A. Morozov, Link polynomial calculus and the AENV conjecture, JHEP 04 (2014) 156 [arXiv: 1309.7984] [INSPIRE].

[146] S. Arthamonov, A. Mironov and A. Morozov, Differential hierarchy and additional grading of knot polynomials, Theor. Math. Phys. 179 (2014) 509 [arXiv:1306.5682] [INSPIRE].

[147] S. Gukov, S. Nawata, I. Saberi, M. Stošić and P. Sułkowski, Sequencing BPS Spectra, JHEP 03 (2016) 004 [arXiv: 1512.07883] [INSPIRE].

[148] Y. Kononov and A. Morozov, On the defect and stability of differential expansion, JETP Lett. 101 (2015) 831 [Pisma Zh. Eksp. Teor. Fiz. 101 (2015) 931] [arXiv:1504.07146] [INSPIRE].

[149] A. Morozov, Differential expansion and rectangular HOMFLY for the figure eight knot, Nucl. Phys. B 911 (2016) 582 [arXiv:1605.09728] [INSPIRE]. 
[150] A. Morozov, Factorization of differential expansion for antiparallel double-braid knots, JHEP 09 (2016) 135 [arXiv: 1606. 06015] [INSPIRE].

[151] I. Tuba and H. Wenzl, Representations of the braid group $B_{3}$ and of $\operatorname{SL}(2, \mathbf{Z})$, math/9912013.

[152] S. Nawata, P. Ramadevi and Zodinmawia, Multiplicity-free quantum $6 j$-symbols for $U_{q}\left(\mathfrak{s l}_{N}\right)$, Lett. Math. Phys. 103 (2013) 1389 [arXiv: 1302.5143] [INSPIRE].

[153] J. Gu and H. Jockers, A note on colored HOMFLY polynomials for hyperbolic knots from WZW models, Commun. Math. Phys. 338 (2015) 393 [arXiv: 1407.5643] [INSPIRE].

[154] P. Vogel, The universal Lie algebra, preprint (1999) http://webusers.imj-prg.fr/ pierre.vogel/.

[155] M. Kameyama and S. Nawata, Refined large- $N$ duality for knots, arXiv:1703.05408 [INSPIRE]. 